\title{
ON HYPERSURFACE QUOTIENT SINGULARITIES OF DIMENSION 4
}

\author{
LI CHIANG and SHI-SHYR ROAN
}

Received 18 February 2003

\begin{abstract}
We consider geometrical problems on Gorenstein hypersurface orbifolds of dimension $n \geq 4$ through the theory of Hilbert scheme of group orbits. For a linear special group $G$ acting on $\mathbb{C}^{n}$, we study the $G$-Hilbert scheme $\operatorname{Hilb}^{G}\left(\mathbb{C}^{n}\right)$ and crepant resolutions of $\mathbb{C}^{n} / G$ for $G$ the $A$-type abelian group $A_{r}(n)$. For $n=4$, we obtain the explicit structure of Hilb ${ }^{A_{r}(4)}\left(\mathbb{C}^{4}\right)$. The crepant resolutions of $\mathbb{C}^{4} / A_{r}(4)$ are constructed through their relation with $\operatorname{Hilb}^{A_{r}(4)}\left(\mathbb{C}^{4}\right)$, and the connections between these crepant resolutions are found by the "flop" procedure of 4-folds. We also make some primitive discussion on $\operatorname{Hilb}^{G}\left(\mathbb{C}^{n}\right)$ for $G$ the alternating group $\mathfrak{A}_{n+1}$ of degree $n+1$ with the standard representation on $\mathbb{C}^{n}$; the detailed structure of $\operatorname{Hilb}^{\mathfrak{X}_{4}}\left(\mathbb{C}^{3}\right)$ is explicitly constructed.
\end{abstract}

2000 Mathematics Subject Classification: 14J35, 14J30, 14M25, $20 \mathrm{C} 30$.

1. Introduction. The purpose of this paper is to study some geometrical problems of certain Gorenstein hypersurface orbifolds of dimension 4. The main focus will be on the structure of the newly developed concept of Hilbert scheme of group orbits and its connection with crepant resolutions of the orbifold. For a finite subgroup $G$ in $\mathrm{SL}_{n}(\mathbb{C})$, the $G$-Hilbert scheme $\operatorname{Hilb}^{G}\left(\mathbb{C}^{n}\right)$ was first introduced by Nakamura et al. [6, 8, 9, 14]; one primary goal is to provide a conceptual understanding of crepant resolutions of $\mathbb{C}^{n} / G$ for $n=3$, whose solution was previously known by a computational method, relying heavily on the Miller-Blichfeldt-Dickson classification of finite groups in $\mathrm{SL}_{3}(\mathbb{C})$ [12] and the invariant theory of two simple groups, $I_{60}$ (icosahedral group) and $H_{168}$ (Klein group) [11] (for the existence of crepant resolutions, see [19] and the references therein). For $n=2, \operatorname{Hilb}^{G}\left(\mathbb{C}^{2}\right)$ is the minimal resolution of $\mathbb{C}^{2} / G$, hence it has the trivial canonical bundle $[8,9,14]$. For $n=3$, it was expected that $\operatorname{Hilb}^{G}\left(\mathbb{C}^{3}\right)$ is a crepant resolution of $\mathbb{C}^{3} / G$. Recently, the affirmative answer was obtained, in $[7,15]$, for the abelian group $G$ by techniques in toric geometry, and, in [2], for a general group $G$ by derived category methods bypassing the geometrical analysis of the $G$-Hilbert scheme. With these successful results in dimension 3, a question naturally arises on the possible role of the $G$-Hilbert scheme in crepant resolution problems of orbifolds in dimension $n \geq 4$. For $n \geq 4$, it is a well-known fact that $\mathbb{C}^{n} / G$ might have no crepant resolutions at all, even for a cyclic group $G$ and $n=4$ (for a selection of examples, see, e.g., [17]). To avoid many such complicated exceptional cases, we will restrict our attention only to those with hypersurface singularities. In this paper, we will address certain problems on two specific types of hypersurface Gorenstein quotient singularity $\mathbb{C}^{n} / G$ of dimension $n$; one is the abelian group $G=A_{r}(n)$ defined in (2.19), and the other, group $G$, is the alternating group $\mathfrak{A}_{n+1}$ of degree $n+1$ acting on $\mathbb{C}^{n}$ through the standard 
representation. In the case $G=A_{r}(n), \operatorname{Hilb}^{G}\left(\mathbb{C}^{n}\right)$ is a toric variety, hence the methods for toric geometry provide an effective tool to study its structure. For $n=4$, we will give a detailed derivation of the smooth toric structure of $\mathrm{Hilb}^{A_{r}(4)}\left(\mathbb{C}^{4}\right)$ and construct the crepant toric resolutions of $\mathbb{C}^{4} / A_{r}(4)$ by blowing down the canonical divisors of $\operatorname{Hilb}^{A_{r}(4)}\left(\mathbb{C}^{4}\right)$; in due course, the "flop" of 4 -folds naturally arises in the process (see Theorems 3.4, 3.5, and 4.1, whose statements were previously announced in [3]). We would expect that the concept which appeared in the proof of these theorems will inspire certain clues to other cases, not only the $A_{r}(n)$-type groups, but also nonabelian groups $\mathfrak{A}_{n+1}$ (which are simple groups for $n \geq 4$ ). Group $\mathfrak{A}_{4}$ is a solvable group of order 12 , also called the ternary trihedral group. The crepant resolution of $\mathbb{C}^{3} / \mathfrak{A}_{4}$ was explicitly constructed in [1], and the structure $\operatorname{Hilb}^{\mathfrak{A}_{4}}\left(\mathbb{C}^{3}\right)$ over the origin orbit of $\mathbb{C}^{3} / \mathfrak{A}_{4}$ was analyzed in detail in [6]. Through the representation theory of $\mathfrak{A}_{4}$, we will give the direct verification that Hilb ${ }^{\mathfrak{H}_{4}}\left(\mathbb{C}^{3}\right)$ is smooth and a crepant resolution of $\mathbb{C}^{3} / \mathfrak{A}_{4}$. Though the conclusion is known by the general result in [2] using qualitative arguments, the object of our detailed analysis is to reveal that there exist certain common features in determining the structures of $G$-Hilbert schemes for certain abelian and nonabelian groups $G$ by the computational methods, in the hope that the approach could possibly be applied to higher-dimensional cases. With this in mind, we will restrict our attention only to the case $\mathfrak{A}_{4}$, leaving possible generalizations, applications, or implications to future work.

This paper is organized as follows. In Section 2, we will summarize the main features of the $G$-Hilbert scheme of group orbits and the results in toric geometry needed in later discussions. We will also define group $G$ which we will consider in this paper. Sections 3 and 4 will be devoted to the discussion of the structure of $\operatorname{Hilb}^{G}\left(\mathbb{C}^{4}\right)$ and crepant resolutions of $\mathbb{C}^{4} / G$ for $G=A_{r}(4)$. For simpler terminology to express ideas, also for the description of the geometry of the flop of 4-folds, we will consider only the case $A_{1}(4)$ in Section 3 to discuss the structure of $\operatorname{Hilb}^{A_{1}(4)}\left(\mathbb{C}^{4}\right)$. The flop relation between crepant resolutions of $\mathbb{C}^{4} / A_{1}(4)$ will be examined in detail through $\operatorname{Hilb}^{A_{1}(4)}\left(\mathbb{C}^{4}\right)$. In Section 4, we will derive the solution of the corresponding problems for $G=A_{r}(4)$ for a general positive integer $r$, with much more complicated techniques but a method much in tune with the previous section. In Section 5 , we consider the case $G=\mathfrak{A}_{n+1}$ acting on $\mathbb{C}^{n}$ through the standard representation for $n=3$. By employing the structure of the fiber in $\mathrm{Hilb}^{\mathfrak{A}_{4}}\left(\mathbb{C}^{3}\right)$ over the origin orbit of $\mathbb{C}^{3} / \mathfrak{A}_{4}$ as described in [6], we give an explicit construction of the smooth and crepant structure of $\operatorname{Hilb}^{\mathfrak{X}_{4}}\left(\mathbb{C}^{3}\right)$ using finite group representation theory, along a line similar to the previous two sections in a certain sense. Finally, we give the concluding remarks in Section 6.

Notations 1.1. To present our work, we prepare some notations. In this paper, by an orbifold we will always mean the orbit space of a smooth complex manifold acted on by a finite group. Throughout the paper, $G$ will always denote a finite group unless otherwise stated. We denote

$$
\operatorname{Irr}(G)=\left\{\rho: G \longrightarrow \mathrm{GL}\left(V_{\rho}\right) \text { an irreducible representation of } G\right\} .
$$

The trivial representation of $G$ will be denoted by $\mathbf{1}$. For a $G$-module $W$, that is, a $G$ linear representation space $W$, one has the canonical irreducible decomposition 
$W=\bigoplus_{\rho \in \operatorname{Irr}(G)} W_{\rho}$, where $W_{\rho}$ is a $G$-submodule of $W$, isomorphic to $V_{\rho} \otimes W_{\rho}^{0}$ for some trivial $G$-module $W_{\rho}^{0}$. For an analytic variety $X$, we will not distinguish the notions of vector bundle and locally free $O_{X}$-sheaf over $X$.

2. $G$-Hilbert scheme and toric geometry. In this section, we briefly review some basic facts on $\mathrm{Hilb}^{G}\left(\mathbb{C}^{n}\right)$ (the Hilbert scheme of $G$-orbits) and toric geometry necessary for later use, then specify groups $G$ for the discussions of the rest of the sections of this paper.

First, we will always assume $G$ to be a finite subgroup of $\mathrm{SL}_{n}(\mathbb{C})$. Denote $S_{G}:=\mathbb{C}^{n} / G$ with the canonical projection, $\pi_{G}: \mathbb{C}^{n} \rightarrow S_{G}$, and $o:=\pi_{G}(\overrightarrow{0})$. As $G$ acts on $\mathbb{C}^{n}$ freely outside a finite collection of linear subspaces with codimension greater than or equal to $2, S_{G}$ is an orbifold with nonempty singular set $\operatorname{Sing}\left(S_{G}\right)$ of codimension greater than or equal to 2. In fact, the element $o$ is a singular point of $S_{G}$. By a variety $X$ birational over $S_{G}$, we will always mean a proper birational morphism $\sigma$ from $X$ to $S_{G}$ which is biregular between $X \backslash \sigma^{-1}\left(\operatorname{Sing}\left(S_{G}\right)\right)$ and $S_{G} \backslash \operatorname{Sing}\left(S_{G}\right)$,

$$
\sigma: X \rightarrow S_{G}
$$

One can form the commutative diagram via the birational morphism $\sigma$ as follows:

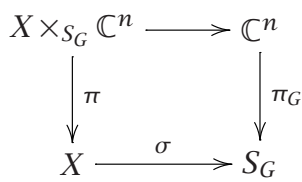

Denote by $\mathscr{F}_{X}$ the coherent $0_{X}$-sheaf over $X$ obtained by the pushforward of the structure sheaf of $X \times_{S_{G}} \mathbb{C}^{n}, \mathscr{F}_{X}:=\pi_{*} O_{X \times S_{G}} \mathbb{C}^{n}$. For two varieties $X, X^{\prime}$ birational over $S_{G}$ with the commutative diagram

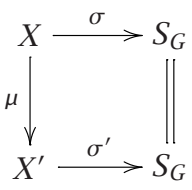

one has a canonical morphism $\mu^{* \mathscr{F}_{X}} \rightarrow \mathscr{F}_{X}$. In particular, the morphism (2.1) gives rise to the $\mathrm{O}_{X}$-morphism

$$
\sigma^{*}\left(\pi_{G *} \mathscr{O}_{\mathbb{C}}\right) \longrightarrow \mathscr{F}_{X}
$$

Furthermore, all the above morphisms are compatible with the natural $G$-structure of $\mathscr{F}_{X}$ induced from the $G$-action on $\mathbb{C}^{n}$ via (2.2). Then $\mathscr{F}_{X}$ has the canonical $G$-decomposition of coherent $\mathscr{O}_{X}$-submodules $\mathscr{F}_{X}=\bigoplus_{\rho \in \operatorname{Irr}(G)}\left(\mathscr{F}_{X}\right)_{\rho}$, where $\left(\mathscr{F}_{X}\right)_{\rho}$ is the $\rho$-factor of $\mathscr{F}_{X}$. The geometrical fibers of $\mathscr{F}_{X}$ and $\left(\mathscr{F}_{X}\right)_{\rho}$ over $x \in X$ are defined by $\mathscr{F}_{X, X}=$ $k(x) \bigotimes_{\mathscr{O}_{X}} \mathscr{F}_{X},\left(\mathscr{F}_{X}\right)_{\rho, x}=k(x) \bigotimes_{\mathscr{O}_{X}}\left(\mathscr{F}_{X}\right)_{\rho}$, where $k(x)\left(:=\mathcal{O}_{X, x} / M_{X}\right)$ is the residue field at $x$. Over $X \backslash \sigma^{-1}\left(\operatorname{Sing}\left(S_{G}\right)\right)$, $\mathscr{F}_{X}$ is a vector bundle of rank $|G|$ with the regular $G$-representation on each geometric fiber. Hence $\left(\mathscr{F}_{X}\right)_{\rho}$ is a vector bundle over $X \backslash \sigma^{-1}\left(\operatorname{Sing}\left(S_{G}\right)\right)$ 
with rank equal to the dimension of $V_{\rho}$. For $x \in X$, there exists a $G$-invariant ideal $I(x)$ in $\mathbb{C}[Z]\left(:=\mathbb{C}\left[Z_{1}, \ldots, Z_{n}\right]\right)$ such that the following relation holds:

$$
\mathscr{F}_{X, x}=k(x) \bigotimes_{{ }^{S_{G}}} \mathbb{O}_{\mathbb{C}}(x) \simeq \mathbb{C}[Z] / I(x)
$$

We have $\left(\mathscr{F}_{X}\right)_{\rho, x} \simeq(\mathbb{C}[Z] / I(x))_{\rho}$. The vector spaces $\mathbb{C}[Z] / I(x)$ form a family of finitedimensional $G$-modules parametrized by $x \in X$. For $x \notin \sigma^{-1}\left(\operatorname{Sing}\left(S_{G}\right)\right), \mathbb{C}[Z] / I(x)$ is a regular $G$-module. In particular, for $X=S_{G}$ in (2.5) and $s \in S_{G}$, the $G$-invariant ideal $I(s)$ of $\mathbb{C}[Z]$ is generated by the $G$-invariant polynomials vanishing at $\sigma^{-1}(s)$. Let $\tilde{I}(s)$ be the ideal of $\mathbb{C}[Z]$ consisting of all polynomials vanishing at $\sigma^{-1}(s)$. Then $\tilde{I}(s)$ is a $G^{-}$ invariant ideal with $\tilde{I}(s) \supset I(s)$. For $s=o$, we have $\tilde{I}(o)=\mathbb{C}[Z]_{0}$ and $I(o)=\mathbb{C}[Z]_{0}^{G} \mathbb{C}[Z]$, where subscript 0 indicates the maximal ideal of polynomials vanishing at the origin. For a variety $X$ birational over $S_{G}$ via $\sigma$ in (2.1), one has the following relations of $G$-invariant ideals of $\mathbb{C}[Z]$ :

$$
\widetilde{I}(s) \supset I(x) \supset I(s), \quad x \in X, s=\sigma(x) .
$$

For $x \in X$, there exists a direct sum decomposition of $\mathbb{C}[Z]$ as $G$-modules,

$$
\mathbb{C}[Z]=I(x)^{\perp} \oplus I(x) .
$$

Here, $I(x)^{\perp}$ is a finite-dimensional $G$-module isomorphic to $\mathbb{C}[Z] / I(x)$. Similarly, we have $G$-module decompositions, for $s=\sigma(x) \in S_{G}$,

$$
\mathbb{C}[Z]=I(s)^{\perp} \oplus I(s), \quad \mathbb{C}[Z]=\widetilde{I}(s)^{\perp} \oplus \widetilde{I}(s),
$$

so that the relations $\tilde{I}(s)^{\perp} \subset I(x)^{\perp} \subset I(s)^{\perp}$ hold. Note that the above finite-dimensional $G$-modules with superscript $\perp$ are not unique in $\mathbb{C}[Z]$ because there is a choice involved, nonetheless, we could choose them such that these inclusions are fulfilled. One has the canonical $G$-decomposition of $I(x)^{\perp}: I(x)^{\perp}=\bigoplus_{\rho \in \operatorname{Irr}(G)} I(x)_{\rho}^{\perp}$, where the factor $I(x)_{\rho}^{\perp}$ is isomorphic to a positive finite sum of copies of $V_{\rho}$.

Now we consider the varieties $X$ birational over $S_{G}$ such that $\mathscr{F}_{X}$ is a vector bundle. Among all such $X$, there exists a minimal object, called the $G$-Hilbert scheme in $[8,9$, $14,15]$,

$$
\sigma_{\text {Hilb }}: \operatorname{Hilb}^{G}\left(\mathbb{C}^{n}\right) \longrightarrow S_{G} \text {. }
$$

By the definition of $\operatorname{Hilb}^{G}\left(\mathbb{C}^{n}\right)$, an element (i.e., closed point) $p$ of $\operatorname{Hilb}^{G}\left(\mathbb{C}^{n}\right)$ is described by a $G$-invariant ideal $I(=I(p))$ of $\mathbb{C}[Z]$ of colength $|G|$, and the fiber of the vector bundle $\mathscr{F}_{\mathrm{Hilb}^{G}\left(\mathbb{C}^{n}\right)}$ over $p$ can be identified with the regular $G$-module $\mathbb{C}[Z] / I(p)$. For simplicity of notation, we will also make the identification of the element $p$ with its associated ideal $I$ and write $I \in \operatorname{Hilb}^{G}\left(\mathbb{C}^{n}\right)$ in what follows if no confusion arises. For any other $X$, map (2.1) can be factored through a birational morphism $\lambda$ from $X$ onto 
$\operatorname{Hilb}^{G}\left(\mathbb{C}^{n}\right)$ via $\sigma_{\text {Hilb: }}$

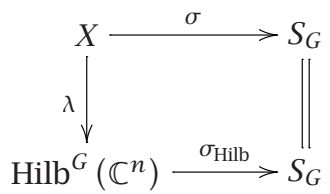

In fact, the ideal $I(x)$ of (2.5) is a colength $|G|$ ideal in $\mathbb{C}[Z]$, by which the map $\lambda$ is defined. We will denote by $X_{G}$ the normalization of $\operatorname{Hilb}^{G}\left(\mathbb{C}^{n}\right)$, which is a normal variety over $S_{G}$ with the birational morphism from $X_{G}$ onto $S_{G}$. As every biregular automorphism of $S_{G}$ can always be lifted to one of $\operatorname{Hilb}^{G}\left(\mathbb{C}^{n}\right)$, hence also to $X_{G}$, one has the following result.

LEMmA 2.1. Let Aut $\left(S_{G}\right)$ be the group of biregular automorphisms of $S_{G}$. Then $\operatorname{Hilb}^{G}\left(\mathbb{C}^{n}\right), X_{G}$ are $\operatorname{Aut}\left(S_{G}\right)$-varieties over $S_{G}$ via $\operatorname{Aut}\left(S_{G}\right)$-morphisms. As a consequence, $X_{G}$ is a toric variety for an abelian group $G$.

Now we are going to summarize some basic facts in toric geometry for the later discussion when group $G$ is abelian (for details, see, e.g., [5, 10, 16]). In this case, we consider $G$ as a subgroup of the diagonal group $T_{0}$ of $\mathrm{GL}_{n}\left(\mathbb{C}^{n}\right)$ with the identification $T_{0}=\mathbb{C}^{* n}$. Regard $\mathbb{C}^{n}$ as the partial compactification of $T_{0}$,

$$
G \subset T_{0} \subset \mathbb{C}^{n} .
$$

Let $T$ be the torus $T_{0} / G$ and consider $S_{G}\left(=\mathbb{C}^{n} / G\right)$ as a $T$-space,

$$
T:=T_{0} / G, \quad T \subset S_{G} .
$$

The combinatorial data of toric varieties are constructed from the lattices of 1-parameter subgroups and characters of tori $T, T_{0}$ :

$$
\begin{aligned}
& N\left(:=\operatorname{Hom}\left(\mathbb{C}^{*}, T\right)\right) \supset N_{0}\left(:=\operatorname{Hom}\left(\mathbb{C}^{*}, T_{0}\right)\right), \\
& M\left(:=\operatorname{Hom}\left(T, \mathbb{C}^{*}\right)\right) \subset M_{0}\left(:=\operatorname{Hom}\left(T_{0}, \mathbb{C}^{*}\right)\right) .
\end{aligned}
$$

For convenience, $N_{0}$ and $N$ will be identified with the following lattices in $\mathbb{R}^{n}$ in this paper. Denote by $\left\{e^{i}\right\}_{i=1}^{n}$ the standard basis of $\mathbb{R}^{n}$, and define the map exp: $\mathbb{R}^{n} \rightarrow T_{0}$ by $r\left(=\sum_{i=1}^{n} r_{i} e^{i}\right) \mapsto \exp (r):=\sum_{i} e^{2 \pi \sqrt{-1}} r_{i} e^{i}$. The lattices $N, N_{0}$ are given by

$$
N_{0}=\mathbb{Z}^{n}\left(:=\exp ^{-1}(1)\right), \quad N=\exp ^{-1}(G)
$$

and we have $G \simeq N / N_{0}$. The lattice $M_{0}$ dual to $N_{0}$ is the standard one in the dual space $\mathbb{R}^{n *}$. In what follows, we will identify $M_{0}$ with the group of monomials in variables $Z_{1}, \ldots, Z_{n}$ via the correspondence

$$
I=\sum_{s=1}^{n} i^{s} e_{s} \in M_{0} \longleftrightarrow Z^{I}=\prod_{s=1}^{n} Z_{s}^{i_{s}} .
$$

The dual lattice $M$ of $N$ is the sublattice of $M_{0}$, consisting of all $G$-invariant monomials. Among the varieties $X$ birational over the $T$-space $S_{G}$, we will consider only those $X$ 
with a $T$-structure. It has been known that these toric varieties $X$ are represented by certain combinatorial data in toric geometry. A toric variety over $S_{G}$ is described by a fan $\Sigma=\left\{\sigma_{\alpha} \mid \sigma \in I\right\}$ with the first quadrant of $\mathbb{R}^{n}$ as its support, that is, a rational convex cone decomposition of the first quadrant in $\mathbb{R}^{n}$. Equivalently, these combinatorial data can also be described by the intersection of the fan and the standard simplex $\Delta$ in the first quadrant:

$$
\triangle:=\left\{r \in \mathbb{R}^{n} \mid \sum_{i} r_{i}=1, r_{j} \geq 0 \forall j\right\}
$$

The corresponding data in $\triangle$ are denoted by $\Lambda=\left\{\triangle_{\alpha} \mid \alpha \in I\right\}$ with $\triangle_{\alpha}:=\sigma_{\alpha} \cap \triangle$. Then $\Lambda$ is a polytope decomposition of $\triangle$ with vertices in $\triangle \cap \mathbb{Q}^{n}$. Note that for $\sigma_{\alpha}=\{\overrightarrow{0}\}$, we have $\triangle_{\alpha}=\varnothing$. Such $\Lambda$ will be called a rational polytope decomposition of $\triangle$, and we will denote by $X_{\Lambda}$ the toric variety corresponding to $\Lambda$. If all vertices of $\Lambda$ are in $N, \Lambda$ is called an integral polytope decomposition of $\triangle$. For a rational polytope decomposition $\Lambda$ of $\triangle$, we define $\Lambda(i):=\left\{\Delta_{\alpha} \in \Lambda \mid \operatorname{dim}\left(\Delta_{\alpha}\right)=i\right\}$ for $-1 \leq i \leq n-1$ (here, $\operatorname{dim}(\varnothing):=-1$ ). The $T$-orbits in $X_{\Lambda}$ are parametrized by $\bigsqcup_{i=-1}^{n-1} \Lambda(i)$. In fact, to $\triangle_{\alpha} \in \Lambda(i)$ there associates an $(n-1-i)$-dimensional $T$-orbit, which will be denoted by orb $\left(\triangle_{\alpha}\right)$. A toric divisor in $X_{\Lambda}$ is the closure of an $(n-1)$-dimensional orbit, denoted by $D_{v}=\overline{\operatorname{orb}(v)}$ for $v \in \Lambda(0)$. The canonical sheaf of $X_{\Lambda}$ is expressed by the toric divisors (see, e.g., $[5,10,16]$ )

$$
\omega_{X_{\Lambda}}=O_{X_{\Lambda}}\left(\sum_{v \in \Lambda(0)}\left(m_{v}-1\right) D_{v}\right),
$$

where $m_{v}$ is the least positive integer with $m_{v} v \in N$. In particular, $X_{\Lambda}$ is crepant, that is, $\omega_{X_{\Lambda}}=O_{X_{\Lambda}}$, if and only if $\Lambda$ is integral. On the other hand, the smoothness of $X_{\Lambda}$ is described by the decomposition $\Lambda$ to be a simplicial one with the multiplicity one property, that is, for each $\Lambda_{\alpha} \in \Delta(n-1)$, the elements $m_{v} v$ for $v \in \Lambda_{\alpha} \cap \Lambda(0)$ form a $\mathbb{Z}$-basis of $N$. The following results are known for toric variety over $S_{G}$ (see, e.g., [18]):

(1) the Euler number of $X_{\Lambda}$ is given by $\chi\left(X_{\Lambda}\right)=|\Lambda(n-1)|$;

(2) for a rational polytope decomposition $\Lambda$ of $\Delta$, any two of the following three properties imply the third one:

$$
X_{\Lambda} \text { : nonsingular, } \quad \omega_{X_{\Lambda}}=\mathcal{O}_{X_{\Lambda}}, \quad \chi\left(X_{\Lambda}\right)=|G| .
$$

In this paper, we will consider only two specific series of hypersurface $n$-orbifold $S_{G}$ for $n \geq 2$. The first type can be regarded as a generalization of the $A$-type Klein surface singularity; the group $G$ is defined as follows:

$$
A_{r}(n):=\left\{g \in \mathrm{SL}_{n}(\mathbb{C}) \mid g: \text { diagonal, } g^{r+1}=1\right\}, \quad r \geq 1 .
$$

The $A_{r}(n)$-invariant polynomials in $\mathbb{C}[Z]\left(:=\mathbb{C}\left[Z_{1}, \ldots, Z_{n}\right]\right)$ are generated by monomials, $X:=\prod_{i=1}^{n} Z_{i}$ and $Y_{j}:=Z_{j}^{r+1}(j=1, \ldots, n)$. Thus $S_{A_{r}(n)}$ is realized as the hypersurface in $\mathbb{C}^{n+1}$

$$
S_{A_{r}(n)}: x^{r+1}=\prod_{j=1}^{n} y_{j}, \quad\left(x, y_{1}, \ldots, y_{n}\right) \in \mathbb{C}^{n+1}
$$


The ideal $I(o)$ of $\mathbb{C}[Z]$ for the element $o \in S_{A_{r}(n)}$ is given by $I(o)=\left\langle Z_{1}^{r+1}, \ldots, Z_{n}^{r+1}\right.$, $\left.Z_{1}, \ldots, Z_{n}\right\rangle$, hence

$$
I(o)^{\perp}=\bigoplus\left\{\mathbb{C} Z^{I} \mid I=\left(i^{1}, \ldots, i^{n}\right), 0 \leq i^{j} \leq r, \prod_{j=1}^{n} i^{j}=0\right\} .
$$

For a nontrivial character $\rho$ of $A_{r}(n)$, the dimension of $I(o)_{\rho}^{\perp}$ is always greater than one. In fact, one can describe an explicit set of monomial generators of $I(o) \stackrel{\perp}{\rho}$. For example, say $I(o) \stackrel{\perp}{\rho}$ contains an element $Z^{I}$ with $I=\left(i^{1}, \ldots, i^{n}\right), i^{1}=0$ and $i^{s} \leq i^{s+1}$, then $I(o)_{\rho}^{\perp}$ is generated by $Z^{K}$ 's with $K=\left(k^{1}, \ldots, k^{n}\right)$ given by

$$
k^{s}= \begin{cases}r+1-i^{j}+i^{s} & \text { if } i^{s}<i^{j} \\ i^{s}-i^{j} & \text { otherwise }\end{cases}
$$

here, $j$ runs through 1 to $n$. Note that some of the above $n$-tuples $K$ might coincide with each other. In particular, for $r=1$, the dimension of $I(o)_{\rho}^{\perp}$ is equal to 2 for $\rho \neq \mathbf{1}$, with a basis consisting of $Z^{I}, Z^{I^{\prime}}$ whose indices satisfy the relations $0 \leq i^{s}, i^{s^{\prime}} \leq 1, i^{s}+i^{s^{\prime}}=1$ for $1 \leq s \leq n$.

The second type of group $G$ is the alternating group $\mathfrak{A}_{n+1}$ (of degree $n+1$ ) acting on $\mathbb{C}^{n}$ through the standard representation. The representation is induced from the linear action of the symmetric group $\Im_{n+1}$ on $\mathbb{C}^{n+1}$ by permuting the coordinate indices, then restricting on the subspace

$$
V=\left\{\left(\tilde{z}_{1}, \ldots, \tilde{z}_{n+1}\right) \in \mathbb{C}^{n+1} \mid \sum_{j=1}^{n+1} \tilde{z}_{j}=0\right\} \simeq \mathbb{C}^{n}
$$

We denote by $\mathbb{C}[\tilde{Z}]\left(:=\mathbb{C}\left[\tilde{Z}_{1}, \ldots, \tilde{Z}_{n+1}\right]\right)$ the coordinate ring of the affine $(n+1)$-space $\mathbb{C}^{n+1}$, and their elementary symmetric polynomials $\sigma_{k}:=\sum_{1 \leq i_{1}<\cdots<i_{k} \leq n+1} \tilde{Z}_{i_{1}} \cdots \tilde{Z}_{i_{k}}$ for $1 \leq k \leq n+1$. The $\mathfrak{A}_{n+1}$-invariant polynomials in $\mathbb{C}[\tilde{Z}]$ are generated by the above $\sigma_{k}$ 's and $\delta:=\prod_{i<j}\left(\tilde{Z}_{i}-\tilde{Z}_{j}\right)$ with a relation $\delta^{2}=\tilde{F}\left(\sigma_{1}, \sigma_{2}, \ldots, \sigma_{n+1}\right)$ for a certain polynomial $\widetilde{F}$. In fact, $\widetilde{F}$ is a (quasi-)homogeneous polynomial of degree $n(n+1)$ with the weights of $\sigma_{k}$ and $\delta$ equal to $k$ and $n(n+1) / 2$, respectively. Denote by $s_{k}, d$ the restriction functions of $\sigma_{k}, \delta$, respectively, on $V$. Then $s_{1}$ is the zero function and $V / \Im_{n+1}=\mathbb{C}^{n}$ via the coordinates $\left(s_{2}, \ldots, s_{n+1}\right)$. The orbifold $S_{\mathfrak{A}_{n+1}}\left(=V / \mathfrak{A}_{n+1}\right)$ is a double cover of $\mathbb{C}^{n}$ :

$$
S_{\mathfrak{A}_{n+1}} \longrightarrow \mathbb{C}^{n}=V / \Im_{n+1}
$$

Then $V / \Im_{n+1}$ can be realized as a hypersurface in $\mathbb{C}^{n+1}$ with the equation

$$
S_{\mathcal{A}_{n+1}}: d^{2}=F_{n}\left(s_{2}, \ldots, s_{n+1}\right), \quad\left(d, s_{2}, \ldots, s_{n+1}\right) \in \mathbb{C}^{n+1},
$$


where $F_{n}\left(s_{2}, \ldots, s_{n+1}\right):=\tilde{F}\left(0, s_{2}, \ldots, s_{n+1}\right)$. The polynomial $F_{n}\left(s_{2}, \ldots, s_{n+1}\right)$ has a lengthy expression in general. Here, we list the polynomials $F_{n}$ for $n=3,4$ :

$$
\begin{aligned}
F_{3}\left(s_{2}, s_{3}, s_{4}\right)= & -4 s_{2}^{3} s_{3}^{2}-27 s_{3}^{4}+16 s_{2}^{4} s_{4}-128 s_{2}^{2} s_{4}^{2}+144 s_{2} s_{3}^{2} s_{4}+256 s_{4}^{3}, \\
F_{4}\left(s_{2}, s_{3}, s_{4}, s_{5}\right)= & -4 s_{2}^{3} s_{3}^{2} s_{4}^{2}-27 s_{3}^{4} s_{4}^{2}+16 s_{2}^{4} s_{4}^{3}+144 s_{2} s_{3}^{2} s_{4}^{3}-128 s_{2}^{2} s_{4}^{4} \\
& +256 s_{4}^{5}-72 s_{2}^{4} s_{3} s_{4} s_{5}+108 s_{3}^{5} s_{5}-630 s_{2} s_{3}^{3} s_{4} s_{5}-1600 s_{3} s_{4}^{3} s_{5} \\
& +560 s_{2}^{2} s_{3} s_{4}^{2} s_{5}+16 s_{2}^{3} s_{3}^{3} s_{5}-900 s_{2}^{3} s_{4} s_{5}^{2}+2250 s_{3}^{2} s_{4} s_{5}^{2} \\
& +2000 s_{2} s_{4}^{2} s_{5}^{2}+108 s_{2}^{5} s_{5}^{2}+825 s_{2}^{2} s_{3}^{2} s_{5}^{2}-3750 s_{2} s_{3} s_{5}^{3}+3125 s_{5}^{4} .
\end{aligned}
$$

3. $A_{1}(4)$-singularity and flop of 4 -folds. We now study the $A_{1}(n)$-singularity with $n \geq 4$. The set of $N$-integral elements in $\Delta$ is given by

$$
\triangle \cap N=\left\{e^{j} \mid 1 \leq j \leq n\right\} \cup\left\{v^{i, j} \mid 1 \leq i<j \leq n\right\},
$$

where $v^{i, j}:=(1 / 2)\left(e^{i}+e^{j}\right)$ for $i \neq j$. Other than the simplex $\Delta$ itself, there is only one integral polytope decomposition of $\Delta$ invariant under all permutations of coordinates, and we will denote it by $\Xi$. $\Xi(n-1)$ consists of $n+1$ elements: $\triangle_{i}(1 \leq i \leq n)$ and $\diamond$, where $\triangle_{i}$ is the simplex generated by $e^{i}$ and $v^{i, j}$ for $j \neq i$, and $\diamond$ is the closure of $\triangle \backslash \bigcup_{i=1}^{n} \triangle_{i}$; equivalently, $\diamond$ equals the convex hull spanned by $v^{i, j}$ 's for $i \neq j$. The lower-dimensional polytopes of $\Xi$ are the faces of those in $\Xi(n-1) . X_{\Xi}$ has the trivial canonical sheaf. For $n=2,3, X_{\Xi}$ is a crepant resolution of $S_{A_{1}(n)}$. For $n=4$, one has the following result.

LEMMA 3.1. For $n=4$, the toric variety $X_{\Xi}$ is smooth except for one isolated singularity, which is the 0-dimensional T-orbit corresponding to $\diamond$.

Proof. In general, for $n \geq 4$, it is easy to see that for each $i$, the vertices of $\Delta_{i}$ form a $\mathbb{Z}$-basis of $N$; for example, say $i=1$, it follows from $\left|A_{1}(n)\right|=2^{n-1}$ and $\operatorname{det}\left(e^{1}, v^{1,2}, \ldots\right.$, $\left.v^{1, n}\right)=1 / 2^{n-1}$. Hence $X_{\Xi}$ is nonsingular near the $T$-orbits associated to simplices in $\Delta_{i}$. As $\diamond$ is not a simplex, orb $(\diamond)$ is always a singular point of $X_{\Xi}$. For $n=4$, the statement of smoothness of $X_{\Xi}$ except orb $(\diamond)$ follows from the fact that for $1 \leq i \leq 4$, the vertices $v^{i, j}(j \neq i)$ of $X_{\Xi}$, together with $(1 / 2) \sum_{j=1}^{4} e^{j}$, form an $N$-basis.

REMARK 3.2. For $n \geq 4$, the following properties hold for 0 -dimensional $T$-orbits of $X_{\Xi}$.

(1) Denote $x_{\Delta_{j}}:=\operatorname{orb}\left(\Delta_{j}\right) \in X_{\Xi}$ for $1 \leq j \leq n$. The inverse of the matrix, spanned by vertices of $\Delta_{j},\left(v^{1, j}, \ldots, v^{j-1, j}, e^{j}, v^{j+1, j}, \ldots, v^{n, j}\right)^{-1}$, gives rise to affine coordinates $\left(U_{1}, \ldots, U_{n}\right)$ centered at $x_{\Delta_{j}}$ such that $U_{i}=Z_{i}^{2}(i \neq j)$ and $U_{j}=Z_{j} / Z_{1} \cdots \check{Z}_{j} \cdots Z_{n}$. Hence $I\left(x_{\Delta_{j}}\right)=\left\langle Z_{j}, Z_{i}^{2}, i \neq j\right\rangle+I(o)$, and we have the regular $A_{1}(n)$-module structure of $\mathbb{C}[Z] / I\left(x_{\Delta_{j}}\right)$ :

$$
\mathbb{C}[Z] / I\left(x_{\Delta_{j}}\right) \simeq \bigoplus\left\{\mathbb{C} Z^{I} \mid I=\left(i_{1}, \ldots, i_{n}\right), i_{j}=0, i_{k}=0,1 \text { for } k \neq j\right\} .
$$

(2) We will denote $x_{\diamond}:=\operatorname{orb}(\diamond)$ in $X_{\Xi}$. The singular structure of $x_{\diamond}$ is determined by the $A_{1}(n)$-invariant polynomials corresponding to the $M$-integral elements in the cone dual to the one generated by $\diamond$ in $N_{\mathbb{R}}$. So the $A_{1}(n)$-invariant polynomials are generated 

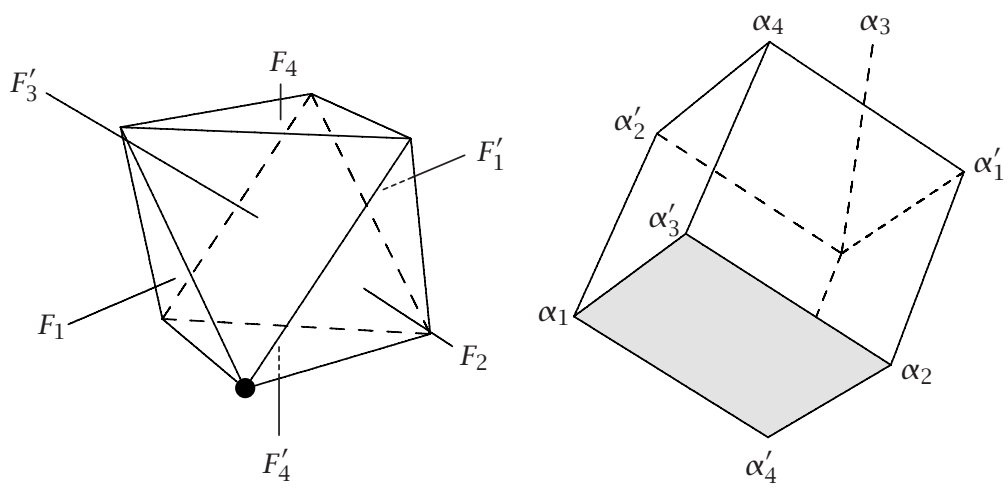

Figure 3.1. Dual pair of octahedron and cube: faces $F_{j}, F_{j}^{\prime}$ of octahedron are dual to vertices $\alpha_{j}, \alpha_{j}^{\prime}$ of cube. The face of the cube in gray color corresponds to the dot "๑" in the octahedron.

by $X_{j}:=Z_{j}^{2}$ and $Y_{j}:=Z_{1} \cdots \check{Z}_{j} \cdots Z_{n} / Z_{j}$. Hence $I\left(x_{\diamond}\right)=\left\langle Z_{1} \cdots \check{Z}_{j} \cdots Z_{n}\right\rangle_{1 \leq j \leq n}+I(o)$. Note that for $n=3$, the $Y_{j}$ 's indeed form the minimal generators for the invariant polynomials, which implies the smoothness of $X_{\Xi}$. For $n \geq 4, x_{\diamond}$ is a singularity not of the hypersurface type. For $n=4$, the $X_{j}, Y_{j}(1 \leq j \leq 4)$ form a minimal set of generators of invariant polynomials, hence the structure near $x_{\diamond}$ in $X_{\Xi}$ is the 4-dimensional affine variety in $\mathbb{C}^{8}$ defined by the relations

$$
x_{i} y_{i}=x_{j} y_{j}, \quad x_{i} x_{j}=y_{i^{\prime}} y_{j^{\prime}}, \quad\left(x_{i}, y_{i}\right)_{1 \leq i \leq 4} \in \mathbb{C}^{8},
$$

where $i \neq j$ with $\left\{i^{\prime}, j^{\prime}\right\}$ the complementary pair of $\{i, j\}$.

For the rest of this section, we will consider only the case $n=4$. We are going to discuss the structure of $\operatorname{Hilb}^{A_{1}(4)}\left(\mathbb{C}^{4}\right)$ and its connection with crepant resolutions of $S_{A_{1}(4)}$. The simplex $\Delta$ is a tetrahedron and $\diamond$ is an octahedron; both are acted on by the symmetric group $\Im_{4}$. The dual polygon of $\diamond$ is the cube. The facets of the octahedron $\diamond$ are labeled by $F_{j}, F_{j}^{\prime}$ for $1 \leq j \leq 4$, where $F_{j}=\diamond \cap \triangle_{j}$ and $F_{j}^{\prime}=\left\{\sum_{i=1}^{4} x_{i} e^{i} \in \diamond \mid x_{j}=0\right\}$. The duals of $F_{j}, F_{j}^{\prime}$ in the cube are vertices, denoted by $\alpha_{j}, \alpha_{j}^{\prime}$ as in Figure 3.1.

Consider the rational simplicial decomposition $\Xi^{*}$ of $\Delta$, which is a refinement of $\Xi$ by adding the center $c:=(1 / 4) \sum_{j=1}^{4} e^{j}$ as a vertex with the barycentric decomposition of $\diamond$ in $\Xi$ (see Figure 3.2). Note that $c \notin N$ and $2 c \in N$. For convenience, we will use the following convention.

NotATION 3.3. Let $G$ be a diagonal group acting on $\mathbb{C}[Z]$. Two monomials $m_{1}, m_{2}$ in $\mathbb{C}[Z]$ are said to be $G$-equivalent, denoted by $m_{1} \stackrel{G}{\sim} m_{2}$ or simply by $m_{1} \sim m_{2}$, if $m_{1} / m_{2}$ is a $G$-invariant function.

THEOREM 3.4. For $G=A_{1}(4)$, $\operatorname{Hilb}^{G}\left(\mathbb{C}^{4}\right) \simeq X_{\Xi *}$, which is nonsingular with the canonical bundle $\omega=O_{X_{\Xi *}}(E)$, where $E$ is an irreducible divisor isomorphic to the triple product 


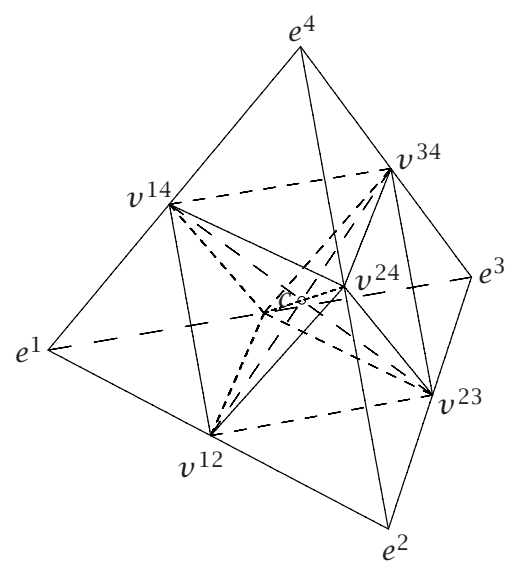

FigURE 3.2. The rational simplicial decomposition $\Xi^{*}$ of $\Delta$ for $n=4, r=1$.

of $\mathbb{P}^{1}$,

$$
E=\mathbb{P}^{1} \times \mathbb{P}^{1} \times \mathbb{P}^{1}
$$

Furthermore, for $\{i, j, k\}=\{1,2,3\}$, the normal bundle of $E$, when restricted on the fiber $\mathbb{P}_{k}^{1}\left(\simeq \mathbb{P}^{1}\right)$, for the projection $E$ to $\mathbb{P}^{1} \times \mathbb{P}^{1}$ via the $(i, j)$ th factor

$$
p_{k}: E \longrightarrow \mathbb{P}^{1} \times \mathbb{P}^{1}
$$

is the (-1)-hyperplane bundle

$$
\mathcal{O}_{X_{\Xi *}}(E) \otimes \mathbb{O}_{\mathbb{P}_{k}^{1}} \simeq \mathcal{O}_{\mathbb{P} 1}(-1)
$$

Proof. First we show the smoothness of the toric variety $X_{\Xi^{*}}$. The octahedron $\diamond$ of $\Xi$ is decomposed into eight simplices of $\Xi^{*}$ corresponding to faces $F_{j}, F_{j}^{\prime}$ of $\diamond$. Denote by $C_{j}$ (resp., $C_{j}^{\prime}$ ) the simplex of $\Xi^{*}$ spanned by $c$ and $F_{j}$ (resp., $F_{j}^{\prime}$ ); $x_{C_{j}}, x_{C_{j}^{\prime}}$ are the corresponding 0-dimensional T-orbits in $X_{\Xi *}$. The smoothness of affine space in $X_{\Xi *}$ near $x_{C_{j}}, x_{C_{j}^{\prime}}$ follows from the $N$-integral criterion of the cones in $N_{\mathbb{R}}$ generated by $C_{j}, C_{j}^{\prime}$. The coordinate system is given by the integral basis of $M$ which generates the cone dual to the cone spanned by $C_{j}\left(C_{j}^{\prime}\right)$. As examples for $C_{1}, C_{2}^{\prime}$, the coordinates are determined by the row vectors of the following square matrices:

$$
\begin{aligned}
\text { cone }\left(C_{1}\right)^{*}: & \left(2 c, v^{1,2}, v^{1,3}, v^{1,4}\right)^{-1}=\left(\begin{array}{cccc}
-1 & 1 & 1 & 1 \\
1 & 1 & -1 & -1 \\
1 & -1 & 1 & -1 \\
1 & -1 & -1 & 1
\end{array}\right), \\
\text { cone }\left(C_{2}^{\prime}\right)^{*}: & \left(v^{3,4}, 2 c, v^{1,4}, v^{1,3}\right)^{-1}=\left(\begin{array}{cccc}
-1 & -1 & 1 & 1 \\
0 & 2 & 0 & 0 \\
1 & -1 & -1 & 1 \\
1 & -1 & 1 & -1
\end{array}\right) .
\end{aligned}
$$


The coordinate functions of $X_{\Xi *}$ centered at $x_{C_{1}}$ are given by $\left(U_{1}, U_{2}, U_{3}, U_{4}\right)=\left(Z_{2} Z_{3} Z_{4} /\right.$ $\left.Z_{1}, Z_{1} Z_{2} / Z_{3} Z_{4}, Z_{1} Z_{3} / Z_{2} Z_{4}, Z_{1} Z_{4} / Z_{2} Z_{3}\right)$ with $I\left(x_{C_{1}}\right)=\left\langle Z_{2} Z_{3} Z_{4}, Z_{1} Z_{2}, Z_{1} Z_{3}, Z_{1} Z_{4}\right\rangle+I(o)$, and the coordinates near $x_{C_{2}^{\prime}}$ are $\left(U_{1}^{\prime}, U_{2}^{\prime}, U_{3}^{\prime}, U_{4}^{\prime}\right)=\left(Z_{3} Z_{4} / Z_{1} Z_{2}, Z_{2}^{2}, Z_{1} Z_{4} / Z_{2} Z_{3}, Z_{1} Z_{3} /\right.$ $\left.Z_{2} Z_{4}\right)$ with $I\left(x_{C_{2}^{\prime}}\right)=\left\langle Z_{3} Z_{4}, Z_{2}^{2}, Z_{1} Z_{4}, Z_{1} Z_{3}\right\rangle+I(o)$. By Remark 3.2(1), one has the smooth coordinate system centered at $x_{\Delta_{j}}$ in $X_{\Xi *}$. For $\Delta_{1}$, by

$$
\text { cone }\left(\Delta_{1}\right)^{*}: \quad\left(e^{1}, v^{1,2}, v^{1,3}, v^{1,4}\right)^{-1}=\left(\begin{array}{cccc}
1 & -1 & -1 & -1 \\
0 & 2 & 0 & 0 \\
0 & 0 & 2 & 0 \\
0 & 0 & 0 & 2
\end{array}\right) \text {, }
$$

one has the coordinate system near $x_{\Delta_{1}},\left(V_{1}, V_{2}, V_{3}, V_{4}\right)=\left(Z_{1} / Z_{2} Z_{3} Z_{4}, Z_{2}^{2}, Z_{3}^{2}, Z_{4}^{2}\right)$ with $I\left(x_{\Delta_{1}}\right)=\left\langle Z_{1}, Z_{2}^{2}, Z_{3}^{2}, Z_{4}^{2}\right\rangle+I(o)$. Now we are going to show that $\mathbb{C}[Z] / I(y)$ is a regular $G$-module for $y \in X_{\Xi *}$. For an element $y$ in the affine neighborhood of $x_{\Delta_{1}}$ with the coordinates $V_{i}=v_{i}(1 \leq i \leq 4)$, one has

$$
I(y)=\left\langle Z_{1}-v_{1} Z_{2} Z_{3} Z_{4}, Z_{2}^{2}-v_{2}, Z_{3}^{2}-v_{3}, Z_{4}^{2}-v_{4}\right\rangle .
$$

The set of monomials $\left\{1, Z_{2}, Z_{3}, Z_{4}, Z_{2} Z_{3}, Z_{2} Z_{4}, Z_{3} Z_{4}, Z_{2} Z_{3} Z_{4}\right\}$ gives rise to a basis of $\mathbb{C}[Z] / I(y)$ for $v_{i} \in \mathbb{C}$; hence $\mathbb{C}[Z] / I(y)$ is a regular $G$-module. For $y$ near $x_{C_{1}}$ with the coordinates $U_{i}=u_{i}(1 \leq i \leq 4)$, we have

$$
I(y)=\left\langle Z_{2} Z_{3} Z_{4}-u_{1} Z_{1}, Z_{1} Z_{2}-u_{2} Z_{3} Z_{4}, Z_{1} Z_{3}-u_{3} Z_{2} Z_{4}, Z_{1} Z_{4}-u_{4} Z_{2} Z_{3}\right\rangle+I_{G}(y),
$$

where $I_{G}(y)=\left\langle Z_{1} Z_{2} Z_{3} Z_{4}-u_{1}^{2} u_{2} u_{3} u_{4}, Z_{1}^{2}-u_{1} u_{2} u_{3} u_{4}, Z_{2}^{2}-u_{2} u_{1}, Z_{3}^{2}-u_{3} u_{1}, Z_{4}^{2}-u_{4} u_{1}\right\rangle$. This implies that $\mathbb{C}[Z] / I(y)$ is a regular $G$-module with a basis represented by $\left\{1, Z_{1}, Z_{2}\right.$, $\left.Z_{3}, Z_{4}, Z_{2} Z_{3}, Z_{3} Z_{4}, Z_{2} Z_{4}\right\}$. Similarly, the same conclusion holds for $y$ near $x_{C_{2}^{\prime}}$ with the coordinates $U_{i}^{\prime}=u_{i}^{\prime}(1 \leq i \leq 4)$, in which case we have

$$
I(y)=\left\langle Z_{3} Z_{4}-u_{1}^{\prime} Z_{1} Z_{2}, Z_{1} Z_{4}-u_{3}^{\prime} Z_{2} Z_{3}, Z_{1} Z_{3}-u_{4}^{\prime} Z_{2} Z_{4}\right\rangle+I_{G}(y),
$$

with $I_{G}(y)=\left\langle Z_{1} Z_{2} Z_{3} Z_{4}-u_{2}^{\prime 2} u_{1}^{\prime} u_{3}^{\prime} u_{4}^{\prime}, Z_{2}^{2}-u_{2}^{\prime}, Z_{1}^{2}-u_{2}^{\prime} u_{3}^{\prime} u_{4}^{\prime}, Z_{3}^{2}-u_{1}^{\prime} u_{2}^{\prime} u_{4}^{\prime}, Z_{4}^{2}-u_{1}^{\prime} u_{2}^{\prime} u_{3}^{\prime}\right\rangle$, and a basis of $\mathbb{C}[Z] / I(y)$ represented by $\left\{1, Z_{1}, Z_{2}, Z_{3}, Z_{4}, Z_{1} Z_{2}, Z_{2} Z_{3}, Z_{2} Z_{4}\right\}$. The same argument can equally be applied to all affine charts centered at $x_{\Delta_{j}}, x_{C_{j}}, x_{C_{j}^{\prime}}$. Therefore we obtain a morphism

$$
\lambda: X_{\Xi *} \longrightarrow \operatorname{Hilb}^{G}\left(\mathbb{C}^{4}\right) \quad \text { with } I(\lambda(y))=I(y), y \in X_{\Xi *} .
$$

We are going to show that the above morphism $\lambda$ is an isomorphism by constructing its inverse morphism. Let $y^{\prime}$ be an element of $\operatorname{Hilb}^{G}\left(\mathbb{C}^{4}\right)$, represented by a $G$-invariant ideal $J \subset \mathbb{C}[Z]$ with $\mathbb{C}[Z] / J$ as the regular $G$-module. By Gröbner basis techniques [4], for a given monomial order, there is a monomial ideal $\operatorname{lt}(J)$, consisting of all leading monomials of elements in $J$, such that the monomial base of $\mathbb{C}[Z] / \operatorname{lt}(J)$ also gives rise to a basis of $\mathbb{C}[Z] / J$. By this fact, we will first determine the $G$-invariant monomial ideal $J_{0}$ in $\operatorname{Hilb}^{G}\left(\mathbb{C}^{4}\right)$. For a monomial $I$, we will denote by $I^{\dagger}$ the set of monic monomials not in $I$. Since all nonconstant $G$-invariant monomials are in $J_{0}$, we have $Z_{j}^{2}, Z_{1} Z_{2} Z_{3} Z_{4} \in J_{0}$. 
Hence $J_{0}^{\dagger}$ is contained in the set $\mathscr{B}:=\left\{Z^{I} \mid I=\left(i_{1}, \ldots, i_{4}\right), i_{1} i_{2} i_{3} i_{4}=0, i_{j} \leq 1\right\}$. For a nontrivial character $\rho$ of $G$, the $\rho$-eigenspace of $I(o)^{\perp}$ for the element $o \in S_{G}$ is of dimension 2. This implies that for $m_{1} \in \mathscr{B}$ not equal to 1 , there exists exactly one $m_{2} \in \mathscr{B}$ not equal to $m_{1}$ with $m_{2} \sim m_{1}$. When $J_{0}=I\left(x_{\Delta_{1}}\right), I\left(x_{\Delta_{1}}\right)^{\perp}$ has a monomial basis $W:=I\left(x_{\Delta_{1}}\right)^{\dagger}$ consisting of eight elements $Z_{2}^{i_{2}} Z_{3}^{i_{3}} Z_{4}^{i_{4}}, 0 \leq i_{j} \leq 1$, and they form a basis of the $G$-regular representation. By replacing some monomials in $W$ by the other $G$-equivalent ones in $\mathscr{B}$, one obtains a $G$-regular basis $W^{\prime}$. Denote by $W_{0}$ the set of monic monomials in $\mathbb{C}[Z]$. The $W^{\prime}$ 's satisfying $W_{0} \cdot\left(W_{0}-W^{\prime}\right) \subset\left(W_{0}-W^{\prime}\right)$ are in one-to-one correspondence with monomial ideals $J_{0}$ in $\operatorname{Hilb}^{G}\left(\mathbb{C}^{4}\right)$ by the relation $J_{0}=\left\langle W_{0}-W^{\prime}\right\rangle_{\mathbb{C}}$, hence $W^{\prime}=J_{0}^{\dagger}$. By direct counting, there are twelve such $W^{\prime \prime}$ 's and the corresponding twelve $J_{0}$ 's are exactly those $I\left(x_{\mathfrak{x}}\right)$ for $\varkappa \in \Xi^{*}(3)$. The correspondence between $W^{\prime}$ and $\varkappa$ by the relation $W^{\prime}=I\left(x_{\mathfrak{z}}\right)^{\dagger}$ is given as follows:

$$
\begin{array}{r}
\left\{1, Z_{2}, Z_{3}, Z_{4}, Z_{2} Z_{3}, Z_{2} Z_{4}, Z_{3} Z_{4}, Z_{2} Z_{3} Z_{4}\right\} \longleftrightarrow \Delta_{1}, \\
\left\{1, Z_{1}, Z_{3}, Z_{4}, Z_{1} Z_{4}, Z_{1} Z_{3}, Z_{3} Z_{4}, Z_{1} Z_{3} Z_{4}\right\} \longleftrightarrow \Delta_{2}, \\
\left\{1, Z_{1}, Z_{2}, Z_{4}, Z_{1} Z_{4}, Z_{2} Z_{4}, Z_{1} Z_{2}, Z_{1} Z_{2} Z_{4}\right\} \longleftrightarrow \Delta_{3}, \\
\left\{1, Z_{1}, Z_{2}, Z_{3}, Z_{2} Z_{3}, Z_{1} Z_{3}, Z_{1} Z_{2}, Z_{1} Z_{2} Z_{3}\right\} \longleftrightarrow \Delta_{4}, \\
\left\{1, Z_{1}, Z_{2}, Z_{3}, Z_{4}, Z_{2} Z_{3}, Z_{2} Z_{4}, Z_{3} Z_{4}\right\} \longleftrightarrow C_{1}, \\
\quad\left\{1, Z_{1}, Z_{2}, Z_{3}, Z_{4}, Z_{1} Z_{4}, Z_{1} Z_{3}, Z_{3} Z_{4}\right\} \longleftrightarrow C_{2}, \\
\left\{1, Z_{1}, Z_{2}, Z_{3}, Z_{4}, Z_{1} Z_{4}, Z_{2} Z_{4}, Z_{1} Z_{2}\right\} \longleftrightarrow C_{3}, \\
\quad\left\{1, Z_{1}, Z_{2}, Z_{3}, Z_{4}, Z_{2} Z_{3}, Z_{1} Z_{3}, Z_{1} Z_{2}\right\} \longleftrightarrow C_{4}, \\
\quad\left\{1, Z_{1}, Z_{2}, Z_{3}, Z_{4}, Z_{1} Z_{4}, Z_{1} Z_{3}, Z_{1} Z_{2}\right\} \longleftrightarrow C_{1}^{\prime}, \\
\left\{1, Z_{1}, Z_{2}, Z_{3}, Z_{4}, Z_{2} Z_{3}, Z_{2} Z_{4}, Z_{1} Z_{2}\right\} \longleftrightarrow C_{2}^{\prime}, \\
\left\{1, Z_{1}, Z_{2}, Z_{3}, Z_{4}, Z_{2} Z_{3}, Z_{1} Z_{3}, Z_{3} Z_{4}\right\} \longleftrightarrow C_{3}^{\prime}, \\
\left\{1, Z_{1}, Z_{2}, Z_{3}, Z_{4}, Z_{1} Z_{4}, Z_{2} Z_{4}, Z_{3} Z_{4}\right\} \longleftrightarrow C_{4}^{\prime} .
\end{array}
$$

Now we consider an ideal $J$ in $\mathbb{C}[Z]$ which defines an element of $\operatorname{Hilb}^{G}\left(\mathbb{C}^{4}\right)$. By the Gröbner basis argument, as before, there is a monomial ideal $J_{0}(=\operatorname{lt}(J))$ such that $J_{0}^{\dagger}$ gives rise to a basis of $\mathbb{C}[Z] / J$, and $J_{0}=I\left(x_{\mathfrak{R}}\right)$ for some $\mathfrak{\gtrless} \in \Xi^{*}(3)$. For $p \in \mathbb{C}[Z]$, the element $p+J \in \mathbb{C}[Z] / J$ is uniquely expressed in the form $p+J=\sum_{m \in J_{0}^{\dagger}} \gamma(p)_{m} m+J$, that is, $p-\sum_{m \in J_{0}^{\dagger}} \gamma(p)_{m} m \in J$. In particular, for a monomial $p$ in $\mathbb{C}[Z]$ (i.e., $p \in W_{0}$ ), we have $g$. $\left(p-\sum_{m \in J_{0}^{\dagger}} \gamma(p)_{m} m\right) \in J$ for $g \in G$. This implies that $p-\sum_{m \in J_{0}^{\dagger}} \gamma(p)_{m} \mu_{g}(p)^{-1} \mu_{g}(m) m$ $\in J$, where $\mu_{g}(m), \mu_{g}(p) \in \mathbb{C}^{*}$ are the character values of $g$ on $m, p$, respectively; hence

$$
\sum_{m \in J_{0}^{\dagger}} \gamma(p)_{m}\left[\mu_{g}(p)^{-1} \mu_{g}(m)-1\right] m \in J .
$$

As $J_{0}^{\dagger}$ represents a $G$-regular basis for $\mathbb{C}[Z] / J$, we have $\gamma(p)_{m}\left[\mu_{g}(p)^{-1} \mu_{g}(m)-1\right]=0$ for $p \in W_{0}, m \in J_{0}^{\dagger}$, and $g \in G$. Furthermore, for each $p \in W_{0}$, there exists a unique element, denoted by $p_{J_{0}^{\dagger}}$, in $J_{0}^{\dagger}$ with the property $p \sim p_{J_{0}^{\dagger}}$. Hence, for $m \in J_{0}^{\dagger}, m \neq p_{J_{0}^{\dagger}}$ if and only if $\left[\mu_{g}(p)^{-1} \mu_{g}(m)-1\right] \neq 0$ for some $g \in G$, in which case $\gamma(p)_{m}=0$. Therefore 
$p-\gamma(p)_{J_{0}^{\dagger}} p_{J_{0}^{\dagger}} \in J$ and $J$ is the ideal with the generators

$$
J=\left\langle p-\gamma(p)_{p_{J_{0}^{\dagger}}} p_{J_{0}^{\dagger}} \mid p \in W_{0} \cap J_{0}\right\rangle .
$$

Indeed, in the above expression of $J$, it suffices to consider those $p$ 's which form a minimal set of monomial generators of $J_{0}$. Now we are going to assign an element of $X_{\Xi *}$ for a given $J \in \operatorname{Hilb}^{G}\left(\mathbb{C}^{4}\right)$. If the monomial ideal $J_{0}$ associated to $J$ in our previous discussion is equal to $I\left(x_{C_{1}}\right)$, a minimal set of monomial generators of $J_{0}$ and the basis representative set $J_{0}^{\dagger}$ of $\mathbb{C}[Z] / J$ are given by

$$
\begin{aligned}
J_{0} & =\left\langle Z_{1}^{2}, Z_{2}^{2}, \ldots, Z_{4}^{2}, Z_{1} Z_{2}, Z_{1} Z_{3}, Z_{1} Z_{4}, Z_{2} Z_{3} Z_{4}\right\rangle, \\
J_{0}^{\dagger} & =\left\{1, Z_{1}, Z_{2}, Z_{3}, Z_{4}, Z_{2} Z_{3}, Z_{2} Z_{4}, Z_{3} Z_{4}\right\} .
\end{aligned}
$$

By (3.15), $J$ contains the ideal generated by $p-\gamma(p)_{p_{J_{0}^{\dagger}}} p_{J_{0}^{\dagger}}$ for $p=Z_{i}^{2}, Z_{1} Z_{2}, Z_{1} Z_{3}, Z_{1} Z_{4}$, $Z_{2} Z_{3} Z_{4}$ for $1 \leq i \leq 4$, which has a colength at most 8 in $\mathbb{C}[Z]$. Therefore one obtains

$$
\begin{aligned}
J= & \left\langle Z_{1} Z_{4}-\gamma_{14} Z_{2} Z_{3}, Z_{1} Z_{3}-\gamma_{13} Z_{2} Z_{4}, Z_{1} Z_{2}-\gamma_{12} Z_{3} Z_{4}, Z_{2} Z_{3} Z_{4}-\gamma_{234} Z_{1},\right. \\
& \left.Z_{1}^{2}-\gamma_{1}, Z_{2}^{2}-\gamma_{2}, Z_{3}^{2}-\gamma_{3}, Z_{4}^{2}-\gamma_{4}\right\rangle .
\end{aligned}
$$

Moreover, by

$$
0 \equiv Z_{2}\left(Z_{1}^{2}-\gamma_{1}\right)-Z_{1}\left(Z_{1} Z_{2}-\gamma_{12} Z_{3} Z_{4}\right) \equiv\left(\gamma_{12} \gamma_{13} \gamma_{4}-\gamma_{1}\right) Z_{2}(\bmod J)
$$

and $Z_{2} \in J_{0}{ }^{\dagger}$, one has

$$
\gamma_{1}=\gamma_{12} \gamma_{13} \gamma_{4}
$$

By

$$
0 \equiv Z_{1}\left(Z_{4}^{2}-\gamma_{4}\right)-Z_{4}\left(Z_{1} Z_{4}-\gamma_{14} Z_{2} Z_{3}\right) \equiv\left(\gamma_{14} \gamma_{234}-\gamma_{4}\right) Z_{1}(\bmod J)
$$

one obtains

$$
\gamma_{2}=\gamma_{234} \gamma_{12}
$$

Similarly, one has

$$
\gamma_{3}=\gamma_{234} \gamma_{13}, \quad \gamma_{4}=\gamma_{234} \gamma_{14}
$$

Therefore, all $\gamma_{I}$ 's are expressed as functions of $\gamma_{12}, \gamma_{13}, \gamma_{14}, \gamma_{234}$. This implies $J=I(y)$ for an element $y$ of $X_{\Xi *}$ in the affine neighborhood $x_{C_{1}}$ with the coordinate $\left(U_{i}=u_{i}\right)$ by the relations

$$
u_{1}=\gamma_{234}, \quad u_{2}=\gamma_{12}, \quad u_{3}=\gamma_{13}, \quad u_{4}=\gamma_{14} .
$$

The above $y$ is defined to be the element $\lambda^{-1}(J)$ in $X_{\Xi *}$ for the ideal $J$ under the inverse map of $\lambda$. The method can equally be applied to ideals $J$ associated to another monomial ideal $J_{0}$. 
For $J_{0}=I\left(x_{C_{2}^{\prime}}\right)$, we have

$$
J=\left\langle Z_{1} Z_{3}-\gamma_{13}^{\prime} Z_{2} Z_{4}, Z_{1} Z_{4}-\gamma_{14}^{\prime} Z_{2} Z_{3}, Z_{3} Z_{4}-\gamma_{34}^{\prime} Z_{1} Z_{2}, Z_{1}^{2}-\gamma_{1}^{\prime}, Z_{2}^{2}-\gamma_{2}^{\prime}, Z_{3}^{2}-\gamma_{3}^{\prime}, Z_{4}^{2}-\gamma_{4}^{\prime}\right\rangle .
$$

We claim that the variables $\gamma_{2}^{\prime}, \gamma_{34}^{\prime}, \gamma_{13}^{\prime}, \gamma_{14}^{\prime}$ form a system of coordinates near $I\left(x_{C_{2}^{\prime}}\right)$, that is, all the $\gamma_{I}^{\prime}$ 's can be expressed as certain polynomials of these four values. Indeed, we are going to show that $\gamma_{1}^{\prime}=\gamma_{2}^{\prime} \gamma_{13}^{\prime} \gamma_{14}^{\prime}, \gamma_{3}^{\prime}=\gamma_{2}^{\prime} \gamma_{13}^{\prime} \gamma_{34}^{\prime}$, and $\gamma_{4}^{\prime}=\gamma_{2}^{\prime} \gamma_{14}^{\prime} \gamma_{34}^{\prime}$. (Note that the group $G$ in [15, Section 6.1, page 777] is the $A_{1}(4)$ of Theorem 3.4 in this paper. However, we would consider that the statement in [15] about the singular property of $\operatorname{Hilb}^{G}\left(\mathbb{C}^{4}\right)$, by using the structure of $I\left(\Gamma_{3}\right)(u)$ there, is not correct. Indeed, by identifying $Z_{2}, Z_{3}, Z_{4}, Z_{1}$ with $x, y, z, w$, and $\gamma_{2}^{\prime}, \gamma_{3}^{\prime}, \gamma_{4}^{\prime}, \gamma_{1}^{\prime}, \gamma_{34}^{\prime}, \gamma_{13}^{\prime}, \gamma_{14}^{\prime}$ with $u_{1}, u_{2}, \ldots, u_{7}$, respectively, the ideal $J$ in our discussion corresponds to $I\left(\Gamma_{3}\right)(u)$ in [15]. Then, through the three relations we have obtained here, one can easily verify that all the relations among the $u_{i}$ 's listed in [15, page 778] hold.) By

$$
Z_{1}\left(Z_{1} Z_{4}-\gamma_{14}^{\prime} Z_{2} Z_{3}\right)-Z_{4}\left(Z_{1}^{2}-\gamma_{1}^{\prime}\right)=-\gamma_{14}^{\prime} Z_{1} Z_{2} Z_{3}+\gamma_{1}^{\prime} Z_{4} \in J
$$

one has

$$
Z_{2}\left(-\gamma_{14}^{\prime} Z_{1} Z_{2} Z_{3}+\gamma_{1}^{\prime} Z_{4}\right)+\gamma_{14}^{\prime} Z_{1} Z_{3}\left(Z_{2}^{2}-\gamma_{2}^{\prime}\right)=\gamma_{1}^{\prime} Z_{2} Z_{4}-\gamma_{14}^{\prime} \gamma_{2}^{\prime} Z_{1} Z_{3} \in J,
$$

hence

$$
\left(\gamma_{1}^{\prime} Z_{2} Z_{4}-\gamma_{14}^{\prime} \gamma_{2}^{\prime} Z_{1} Z_{3}\right)+\gamma_{14}^{\prime} \gamma_{2}^{\prime}\left(Z_{1} Z_{3}-\gamma_{13}^{\prime} Z_{2} Z_{4}\right)=\left(\gamma_{1}^{\prime}-\gamma_{2}^{\prime} \gamma_{13}^{\prime} \gamma_{14}^{\prime}\right) Z_{2} Z_{4} \in J .
$$

By the description in (3.13) for $C_{2}^{\prime}, Z_{2} Z_{4}$ is an element in $J_{0}^{\dagger}$, hence it represents a basis element of $\mathbb{C}[Z] / J$. The relation $\left(\gamma_{1}^{\prime}-\gamma_{2}^{\prime} \gamma_{13}^{\prime} \gamma_{14}^{\prime}\right) Z_{2} Z_{4} \in J$ implies

$$
\gamma_{1}^{\prime}-\gamma_{2}^{\prime} \gamma_{13}^{\prime} \gamma_{14}^{\prime}=0 \text {. }
$$

By interchanging the indices 1 and 3 (resp., 1 and 4) in the above derivation and regarding $\gamma_{i j}^{\prime}=\gamma_{j i}^{\prime}$, we obtain $\gamma_{3}^{\prime}=\gamma_{2}^{\prime} \gamma_{13}^{\prime} \gamma_{34}^{\prime}$ (resp., $\gamma_{4}^{\prime}=\gamma_{2}^{\prime} \gamma_{14}^{\prime} \gamma_{34}^{\prime}$ ). Thus, $\gamma_{2}^{\prime}, \gamma_{13}^{\prime}, \gamma_{13}^{\prime}$, and $\gamma_{34}^{\prime}$ form the four independent parameters to describe the ideals $J$ near $J_{0}=I\left(x_{C_{2}^{\prime}}\right)$ with the regular $G$-module $\mathbb{C}[Z] / J$. Therefore $J=I(y)$ for $y$ near $x_{C_{2}^{\prime}}$ with the coordinates $\left(U_{i}^{\prime}=u_{i}^{\prime}\right)$ via the relations

$$
u_{2}^{\prime}=\gamma_{2}^{\prime}, \quad u_{1}^{\prime}=\gamma_{34}^{\prime}, \quad u_{3}^{\prime}=\gamma_{14}^{\prime}, \quad u_{4}^{\prime}=\gamma_{13}^{\prime} .
$$

For $J_{0}=I\left(x_{\Delta_{1}}\right)$, we have $J=\left\langle Z_{1}-\gamma_{1}^{\prime \prime} Z_{2} Z_{3} Z_{4}, Z_{2}^{2}-\gamma_{2}^{\prime \prime}, Z_{3}^{2}-\gamma_{3}^{\prime \prime}, Z_{4}^{2}-\gamma_{4}^{\prime \prime}\right\rangle$. Hence $J=$ $I(y)$ for $y$ near $x_{\Delta_{1}}$ with the coordinates $\left(V_{i}=v_{i}\right)$ and the relations $v_{i}=\gamma_{i}^{\prime \prime}$ for $1 \leq$ $i \leq 4$. The previous discussions of the three cases can be applied to each of the twelve monomial ideals $J_{0}$ 's by a suitable change of indices. Hence one obtains an element $\lambda^{-1}(J)$ in $X_{\Xi *}$ of an ideal $J \in \operatorname{Hilb}^{G}\left(\mathbb{C}^{4}\right)$.

However, one has to verify the correspondence $\lambda^{-1}$ so defined to be a single-valued map, namely, for a given $J$ with two possible choices of $J_{0}$, the elements in $X_{\Xi *}$ assigned to $J$ through the previous procedure through these two $J_{0}$ 's are the same. For 
example, say $J=I\left(y_{1}\right)=I\left(y_{2}\right)$ for $y_{1}$ near $x_{\Delta_{1}}$ with $\left(V_{i}=v_{i}\right)$ and $y_{2}$ near $x_{C_{1}}$ with $\left(U_{i}=u_{i}\right)$. By (3.9) and (3.10), both $Z_{2} Z_{3} Z_{4}-u_{1} Z_{1}$ and $Z_{1}-v_{1} Z_{2} Z_{3} Z_{4}$ are elements in $J$. We claim that $u_{1} \neq 0$. Otherwise, both $Z_{1}$ and $Z_{2} Z_{3} Z_{4}$ are elements in $J$ with the same $G$-character $\kappa$. Then the $\kappa$-eigenspace in $\mathbb{C}[Z] / J$ is the zero space, a contradiction to the regular $G$-module property of $\mathbb{C}[Z] / J$. Hence one has $Z_{1}-u_{1}{ }^{-1} Z_{2} Z_{3} Z_{4} \in J$, hence $\left(v_{1}-u_{1}^{-1}\right) Z_{2} Z_{3} Z_{4} \in J$. As $J=I\left(y_{1}\right)$ with $y_{1}$ near $x_{\Delta_{1}}, Z_{2} Z_{3} Z_{4}$ represents a basis element of $\mathbb{C}[Z] / J$. Hence $v_{1}=u_{1}{ }^{-1}$. By $Z_{1} Z_{2}-u_{2} Z_{3} Z_{4}, Z_{2}^{2}-v_{2} \in J$, one has $v_{2} Z_{1}-u_{2} Z_{2} Z_{3} Z_{4}\left(=\left(Z_{1} Z_{2}-u_{2} Z_{3} Z_{4}\right) Z_{2}-\left(Z_{2}^{2}-v_{2}\right) Z_{1}\right) \in J$. As $Z_{2} Z_{3} Z_{4} \notin J$, one has $u_{2}=0$ if $v_{2}=0$. When $v_{2} \neq 0$, we have $Z_{1}-u_{2} v_{2}{ }^{-1} Z_{2} Z_{3} Z_{4} \in J$, hence

$$
\left(v_{1}-u_{2} v_{2}^{-1}\right) Z_{2} Z_{3} Z_{4} \in J, \quad u_{2}=v_{1} v_{2} .
$$

Using the same argument, one can derive $u_{j}=v_{1} v_{j}$ for $j=2,3,4$. These three relations, together with $u_{1}=v_{1}^{-1}$, imply $y_{1}=y_{2}$ in $X_{\Xi *}$.

For $y_{2}$ near $x_{C_{1}}$ with $\left(U_{i}=u_{i}\right)$ and $y_{3}$ near $x_{C_{2}^{\prime}}$ with $\left(U_{i}^{\prime}=u_{i}^{\prime}\right)$, by (3.10), (3.11), both $Z_{1} Z_{2}-u_{2} Z_{3} Z_{4}$ and $Z_{3} Z_{4}-u_{1}^{\prime} Z_{1} Z_{2}$ are elements in $J$; furthermore, $u_{2}, u_{1}^{\prime}$ are nonzero by the fact that only one of $Z_{1} Z_{2}, Z_{3} Z_{4}$ could be an element of $J$. By an argument similar to the one before, one can show

$$
u_{1}^{\prime}=u_{2}^{-1}, \quad u_{3}=u_{4}^{\prime}, \quad u_{4}=u_{3}^{\prime}
$$

By $Z_{2} Z_{3} Z_{4}-u_{1} Z_{1}, Z_{3} Z_{4}-u_{1}^{\prime} Z_{1} Z_{2}, Z_{2}^{2}-u_{2}^{\prime} \in J$, we have

$$
\left(Z_{2} Z_{3} Z_{4}-u_{1} Z_{1}\right) Z_{2} \equiv\left(u_{1}^{\prime} u_{2}^{\prime}-u_{1}\right) Z_{1} Z_{2} \equiv 0 \bmod J
$$

As $Z_{1} Z_{2}$ represents a basis element of $\mathbb{C}[Z] / J$, one has $u_{1}=u_{1}^{\prime} u_{2}^{\prime}$. The four relations between $u_{i}$ 's and $u_{i}^{\prime}$ 's imply $y_{2}=y_{3}$ in $X_{\Xi *}$. In this way, one can show directly that for a given ideal $J$ with $J=I(y)=I\left(y^{\prime}\right)$ for $y, y^{\prime}$ in $X_{\Xi *}$, the elements $y$ and $y^{\prime}$ are the same by the relations of toric coordinates centered at two distinct $x_{\mathfrak{x}}$ 's. Hence we have obtained a well-defined morphism $\lambda^{-1}$ from $\operatorname{Hilb}^{G}\left(\mathbb{C}^{4}\right)$ to $X_{\Xi *}$, then $\operatorname{Hilb}^{G}\left(\mathbb{C}^{4}\right) \simeq X_{\Xi *}$. By (2.17), the canonical bundle of $X_{\Xi^{*}}$ is given by $\omega=\mathcal{O}_{X_{\Xi *}}(E)$, where $E$ denotes the toric divisor $D_{c}$, which is a 3-dimensional complete toric variety with the toric data described by the star of $c$ in $\Xi^{*}$, represented by the octahedron in Figure 3.1, where the cube represents the toric orbits' structure. Therefore $E$ is isomorphic to the triple product of $\mathbb{P}^{1}$ as in (3.4). The description of the normal bundle of $E$ restricting on each $\mathbb{P}^{1}$-fiber will follow by direct computation in toric geometry. For example, for the fibers over the projection of $E$ onto $\left(\mathbb{P}^{1}\right)^{2}$ corresponding to the 2-convex set spanned by $v^{1,2}, v^{1,3}, v^{3,4}$, and $v^{2,4}$, one can perform the computation as follows. Let $\left(U_{1}, U_{2}, U_{3}, U_{4}\right)$ be the local coordinates near $x_{C_{4}^{\prime}}$ dual to the $N$-basis $\left(2 c, v^{1,2}, v^{1,3}, v^{2,3}\right)$, and let $\left(W_{1}, W_{2}, W_{3}, W_{4}\right)$ be the local coordinates near $x_{C_{1}}$ dual to $\left(2 c, v^{1,2}, v^{1,3}, v^{1,4}\right)$. By $2 c=v^{1,4}+v^{2,3}$, one has the relations $U_{1}=W_{1} W_{4}, U_{4}=W_{4}^{-1}, U_{2}=W_{2}, U_{3}=W_{3}$. This shows that the restriction of the normal bundle of $E$ on each fiber $\mathbb{P}^{1}$ over $\left(U_{2}, U_{3}\right)$-plane is the $(-1)$-hyperplane bundle. 

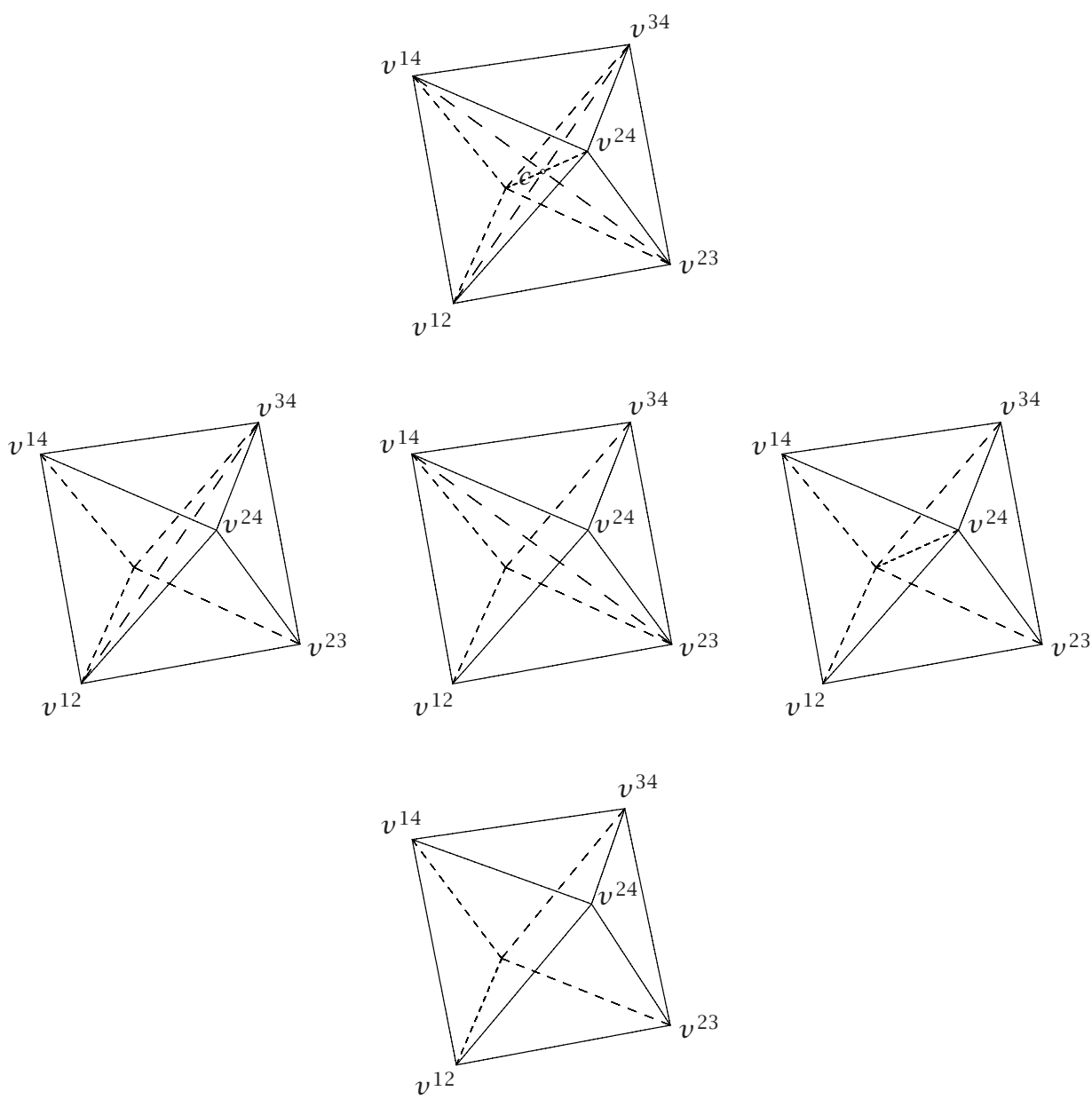

FIGURE 3.3. Toric representation of 4-dimensional flops (in the second row), over a common singular base (in the third row), and dominated by the same 4 -fold (in the first row).

Note that the vector bundle $\mathscr{F}_{X_{\Xi} *}$ over $X_{\Xi *}$ in Theorem 3.4 carries the regular $G$ module structure on each fiber with the local frame of the vector bundle provided by the structure of $\mathbb{C}[Z] / I\left(x_{\mathfrak{R}}\right)$ for $\mathfrak{Z} \in \Xi^{*}$ (3) with the representative in the list (3.13).

By the standard blowing-down criterion of an exceptional divisor, property (3.6) ensures the existence of a smooth 4 -fold $\left(X_{\Xi *}\right)_{k}$ by blowing down the $\mathbb{P}^{1}$-family along the projection $p_{k}$ (3.5) for each $k$. In fact, $\left(X_{\Xi^{*}}\right)_{k}$ is also a toric variety $X_{\Xi_{k}}$ with $\Xi_{k}$ defined by the refinement of $\Xi$ by adding the segment connecting $v^{k, 4}$ and $v^{i, j}$ to divide the central polygon $\diamond$ into 4 simplices, where $\{i, j, k\}=\{1,2,3\}$. Each $X_{\Xi_{k}}$ is a crepant resolution of $X_{\Xi}\left(=S_{G}\right)$, and one has the refinement relation of toric varieties $\Xi \prec \Xi_{k} \prec \Xi^{*}$ for $k=1,2,3$. The polyhedral decomposition in the central core $\diamond$ which appeared in 
the refinements is indicated by the relation

$$
\diamond \prec \diamond_{k} \prec \diamond^{*}, \quad k=1,2,3,
$$

whose pictorial realization is shown in Figure 3.3. The connection between these three smooth 4 -folds corresponding to these different $\diamond_{k}$ 's can be regarded as the "flop" relation of 4-folds, an analogy to the similar procedure in birational geometry of 3folds [13]. Each one is a "small" (here the "smallness," for a resolution, means one with the exceptional locus of codimension greater than or equal to 2) resolution of the 4dimensional isolated singularity with the defining equation (3.3). Hence we have shown the following result.

THEOREM 3.5. For $G=A_{1}(4)$, there are crepant resolutions of $S_{G}$ obtained by blowing down the divisor $\mathrm{E}$ of $\mathrm{Hilb}^{G}\left(\mathbb{C}^{4}\right)$ along (3.5) in Theorem 3.4. Any two such resolutions differ by a "flop" of 4-folds.

4. $G$-Hilbert scheme and crepant resolution of $\mathbb{C}^{4} / A_{r}(4)$. In this section, we give a complete proof of a general result as in Theorem 3.5, but on the group $A_{r}(4)$ for all $r$.

THEOREM 4.1. For $G=A_{r}(4)$, the $G$-Hilbert scheme $\mathrm{Hilb}^{G}\left(\mathbb{C}^{4}\right)$ is a nonsingular toric variety with the canonical bundle $\omega=\mathbb{O}_{\mathrm{Hilb}^{G}\left(\mathbb{C}^{4}\right)}\left(\sum_{k=1}^{m} E_{k}\right)$ with $m=r(r+1)(r+2) / 6$, where $E_{k}$ 's are disjoint smooth exceptional divisors in $\operatorname{Hilb}^{G}\left(\mathbb{C}^{4}\right)$, each of which satisfies conditions (3.4), (3.6). By blowing down $E_{k}$ to $\mathbb{P}^{1} \times \mathbb{P}^{1}$ via a projection (3.5) for each $k$, it gives rise to a toric crepant resolution $\hat{S}_{G}$ of $S_{G}$ with $\chi\left(\hat{S}_{G}\right)=\left|A_{r}(4)\right|=(r+1)^{3}$. Furthermore, any two such $\hat{S}_{G}$ 's differ by a sequence of flops.

Proof. First we define the simplicial decomposition $\Xi^{*}$ of (2.16) for $n=4$, and then we will show that the toric variety $X_{\Xi^{*}}$ is isomorphic to $\mathrm{Hilb}^{G}\left(\mathbb{C}^{4}\right)$. We will denote an element of $N \cap \Delta$ by

$$
\mathbf{v}^{m}\left(=\mathbf{v}^{\left(m_{1}, \ldots, m_{4}\right)^{t}}\right):=\frac{m_{1} e^{1}+m_{2} e^{2}+m_{3} e^{3}+m_{4} e^{4}}{r+1}, \quad 0 \leq m_{i} \leq r+1, \sum_{i=1}^{4} m_{i}=r+1
$$

For each $\mathbf{v}^{m} \in N \cap \Delta$, there are four hyperplanes passing through $\mathbf{v}^{m}$ and parallel to one of the four facets of $\Delta$. The collection of all such hyperplanes gives rise to a polytope decomposition of $\Delta$, denoted by $\Xi$ (for $r=2$, see Figure 4.1(a)).

Now we examine the polytope structure of $\Xi$. We have $\Xi(0)=N \cap \Delta$. For each $\mathbf{v}^{m} \in$ $\Xi(0)$, there are at most twelve segments in $\Xi(1)$ containing $\mathbf{v}^{m}$, and they are given by $\left\langle\mathbf{v}^{m}, \mathbf{v}^{m(i, j)}\right\rangle$ for $i \neq j, 1 \leq i, j \leq 4$, where $m(i, j):=m+e^{i}-e^{j}$. For a given $\left\langle\mathbf{v}^{m}, \mathbf{v}^{m(i, j)}\right\rangle$, the hyperplane passing $\mathbf{v}^{m}$ in $\mathbb{R}^{4}$ with the normal vector $e^{i}-e^{j}$ separates $\Delta$ into two polytopes $\Delta^{\prime}$ s (one of which could possibly be the empty set). We are going to discuss those elements in $\Xi$ containing $\mathbf{v}^{m}$ and lying in a nonempty polytope of these two divided ones. For easier description of our conclusion and for the simplicity of notions, we will work on a special model case, say $i=2, j=3$, and the nonempty polytope $\Delta^{\prime}$ consisting of those elements in $\Delta$ with nonnegative inner product to $e^{2}-e^{3}$ (no difficulties for a similar discussion will arise in other cases except for a suitable change 


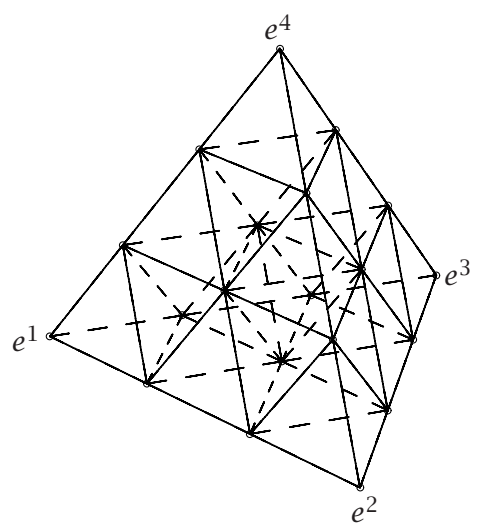

(a)

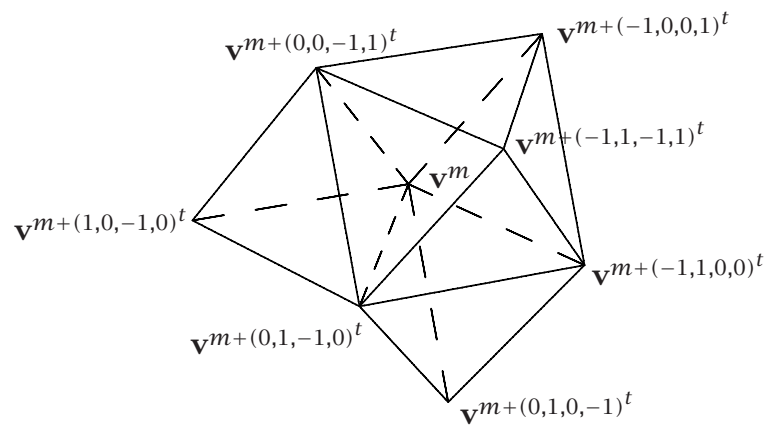

(b)

FIGURE 4.1. The polytope decomposition $\Xi$ of $\Delta$ for $r=2$ and local figure of $\Xi$.

of indices). The elements in $\Xi(3)$ contained in $\Delta^{\prime}$ with $\mathbf{v}^{m}$ as one of its vertices are the following ones:

$$
\begin{gathered}
\Delta_{u}:=\left\langle\mathbf{v}^{m}, \mathbf{v}^{m(2,3)}, \mathbf{v}^{m(1,3)}, \mathbf{v}^{m(4,3)}\right\rangle, \quad \Delta_{d}:=\left\langle\mathbf{v}^{m}, \mathbf{v}^{m(2,3)}, \mathbf{v}^{m(2,1)}, \mathbf{v}^{m(2,4)}\right\rangle, \\
\diamond_{+}:=\left\langle\mathbf{v}^{m}, \mathbf{v}^{m(2,3)}, \mathbf{v}^{m(4,3)}, \mathbf{v}^{m(2,1)}, \mathbf{v}^{m(4,1)}, \mathbf{v}^{m+(-1,1,-1,1)^{t}}\right\rangle, \\
\diamond_{-}:=\left\langle\mathbf{v}^{m}, \mathbf{v}^{m(2,3)}, \mathbf{v}^{m(1,3)}, \mathbf{v}^{m(2,4)}, \mathbf{v}^{m(1,4)}, \mathbf{v}^{m+(1,1,-1,-1)^{t}}\right\rangle .
\end{gathered}
$$

Note that $\diamond_{ \pm}$are similar by interchanging $e^{3}$ and $e^{4}$ (for the configuration of $\Delta_{u}, \Delta_{d}, \diamond_{+}$, see Figure 4.1(b)). Both $\Delta_{u}, \Delta_{d}$ are 3-simplices with their vertices forming an integral basis of $N$, and one facet of each of these 3-simplices is parallel to that of $\Delta$. The toric data of $\Delta_{u}, \Delta_{d}$ give rise to the smooth affine open subsets of $X_{\Xi}$. The polytope $\diamond_{+}$ (resp., $\diamond_{-}$) is an octahedron with the center $c=\mathbf{v}^{m}+\left(e^{2}+e^{4}-e^{1}-e^{3}\right) / 2(r+1)$ (resp., $\left.c=\mathbf{v}^{m}+\left(e^{1}+e^{2}-e^{3}-e^{4}\right) / 2(r+1)\right)$. We will mark the octahedron by its center $c$, and 
denote it by $\diamond^{c}$. The affine open subset of $X_{\Xi}$ with the toric data $\diamond^{c}$ is smooth except for one isolated singular point $x_{\diamond c}$, a 0 -dimensional toric orbit of the affine toric variety. Hence, one can conclude that $\Xi$ (3) consists of three types of elements: $\Delta_{u}, \Delta_{d}$, or $\diamond^{c}$. The toric variety $X_{\Xi}$ is smooth except for the finite number of isolated singularities $x_{\diamond c}$ 's. The structure of $X_{\Xi}$ near a singular element $x_{\diamond c}$ can be determined in the following manner. For a given $\diamond^{c}$, one can construct a tetrahedron $\Delta^{c}$ inside $\Delta$ with the core $\nabla^{c}$ adjacent to four elements $\Delta_{j}^{c}(1 \leq j \leq 4)$ in $\Xi(3)$ of type $\Delta_{u}$ or $\Delta_{d}$,

$$
\Delta^{c}=\diamond^{c} \cup \bigcup_{j=1}^{4} \Delta_{j}^{c} \subseteq \Delta,
$$

such that $\diamond^{c} \cap \Delta_{j}^{c}(1 \leq j \leq 4)$ are four facets of $\diamond^{c}$, two of which intersect only at one common vertex (there could be two possible ways of forming such $\Delta^{c}$ with the same core $\nabla^{c}$ ). Consider the rational simplicial decomposition $\Xi^{*}$ of $\Delta$, which is a refinement of $\Xi$ by adding $c$ as a vertex with the barycentric simplicial decomposition $\diamond^{c}$ for all $c$. In fact, the octahedron $\diamond^{c}$ is decomposed into the following eight 4-simplices of $\Xi^{*}$ :

$$
\begin{aligned}
& C_{1}^{c}:=\left\langle c, c+\frac{e^{1}+e^{2}-e^{3}-e^{4}}{2(r+1)}, c+\frac{e^{1}-e^{2}+e^{3}-e^{4}}{(r+1)}, c+\frac{e^{1}-e^{2}-e^{3}+e^{4}}{2(r+1)}\right\rangle, \\
& C_{2}{ }^{c}:=\left\langle c+\frac{e^{1}+e^{2}-e^{3}-e^{4}}{2(r+1)}, c, c+\frac{-e^{1}+e^{2}+e^{3}-e^{4}}{2(r+1)}, c+\frac{-e^{1}+e^{2}-e^{3}+e^{4}}{2(r+1)}\right\rangle, \\
& C_{3}{ }^{c}:=\left\langle c+\frac{e^{1}-e^{2}+e^{3}-e^{4}}{2(r+1)}, c+\frac{-e^{1}+e^{2}+e^{3}-e^{4}}{2(r+1)}, c, c+\frac{-e^{1}-e^{2}+e^{3}+e^{4}}{2(r+1)}\right\rangle, \\
& C_{4}{ }^{c}:=\left\langle c+\frac{e^{1}-e^{2}-e^{3}+e^{4}}{2(r+1)}, c+\frac{-e^{1}+e^{2}-e^{3}+e^{4}}{2(r+1)}, c+\frac{-e^{1}-e^{2}+e^{3}+e^{4}}{2(r+1)}, c\right\rangle, \\
& C_{1}^{\prime c}:=\left\langle c, c+\frac{-e^{1}-e^{2}+e^{3}+e^{4}}{2(r+1)}, c+\frac{-e^{1}+e^{2}-e^{3}+e^{4}}{2(r+1)}, c+\frac{-e^{1}+e^{2}+e^{3}-e^{4}}{2(r+1)}\right\rangle, \\
& C_{2}^{\prime c}:=\left\langle c+\frac{-e^{1}-e^{2}+e^{3}+e^{4}}{2(r+1)}, c, c+\frac{e^{1}-e^{2}-e^{3}+e^{4}}{2(r+1)}, c+\frac{e^{1}-e^{2}+e^{3}-e^{4}}{2(r+1)}\right\rangle, \\
& C_{3}^{\prime c}:=\left\langle c+\frac{-e^{1}+e^{2}-e^{3}+e^{4}}{2(r+1)}, c+\frac{e^{1}-e^{2}-e^{3}+e^{4}}{2(r+1)}, c, c+\frac{e^{1}+e^{2}-e^{3}-e^{4}}{2(r+1)}\right\rangle, \\
& C_{4}^{\prime c}:=\left\langle c+\frac{-e^{1}+e^{2}+e^{3}-e^{4}}{2(r+1)}, c+\frac{e^{1}-e^{2}+e^{3}-e^{4}}{2(r+1)}, c+\frac{e^{1}+e^{2}-e^{3}-e^{4}}{2(r+1)}, c\right\rangle .
\end{aligned}
$$

All vertices that appeared in the above simplices are elements in $N \cap \Delta$ except $c$, while $2 c \in N$. (See Figure 4.2.)

One can determine the singularity structure of the variety $X_{\Xi}$ near $x_{\diamond c}$ by examining the toric orbits associated to $\Delta^{c}$. The toric data in $\mathbb{R}^{4}$ for the lattice $N$ and the cone generated by $\Delta^{c}$ are isomorphic to the toric data of the lattice for the group $A_{1}$ (4) with the first quadrant cone in Lemma 3.1. Hence, as toric varieties, the structure of $X_{\Xi}$ near the singularity $x_{\diamond c}$ is the same as that of $A_{1}(4)$. One can apply the result of Theorem 4.1 to describe the local structure of $X_{\Xi *}$ over the singular point $x_{\diamond}$ of $X_{\Xi}$. Hence one concludes that $X_{\Xi *}$ is a smooth toric variety with the canonical 


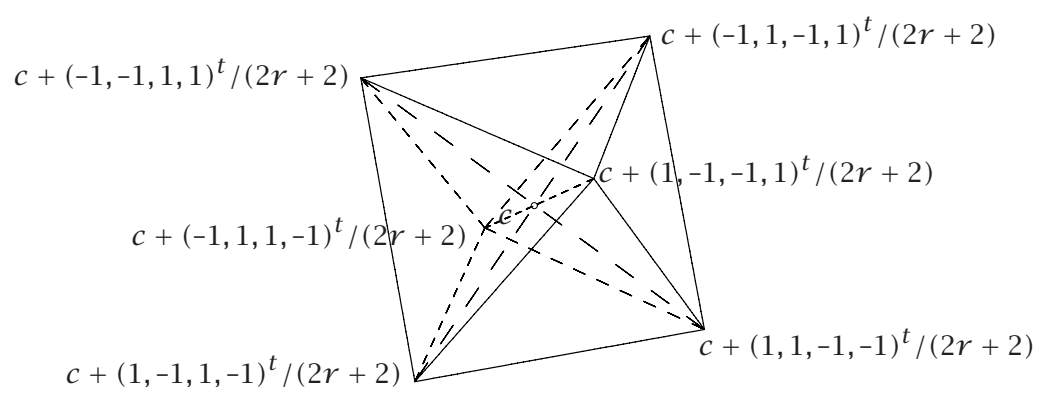

FIGURE 4.2. Local figure of the decomposition of the octahedron in Figure $4.1(\mathrm{~b})$ by adding $c$.

bundle $\omega_{X_{\Xi *}}=\mathscr{O}_{X_{\Xi *} *}\left(\sum_{\diamond c \in \Xi(4)} E_{c}\right)$, where $E_{c}$ is the toric divisor associated to the vertex $c$ in $X_{\Xi *}$, and it satisfies the properties (3.4), (3.6). By (2.18) and the structure of $E_{\mathcal{C}}$, one obtains the desired crepant resolutions $\hat{S}_{A_{r}(4)}$ by blowing down each $E_{\mathcal{c}}$ to $\mathbb{P}^{1} \times \mathbb{P}^{1}$ as in Theorem 3.5, and different crepant resolutions are connected by a flop relation. It remains to show $X_{\Xi^{*}} \simeq \mathrm{Hilb}^{G}\left(\mathbb{C}^{4}\right)$, and the total number of $\diamond^{c}$ 's is equal to $r(r+1)(r+2) / 6$. As in the proof of Theorem 3.4, we first construct a regular morphism $\lambda$ from $X_{\Xi^{*}}$ to $\operatorname{Hilb}^{G}\left(\mathbb{C}^{4}\right)$ by examining $I(y)$, for $y \in X_{\Xi^{*}}$, in terms of toric coordinates. For $\mathfrak{R} \in \Xi^{*}(3)$, we denote $x_{\mathfrak{k}}:=\operatorname{orb}(\mathfrak{k}) \in X_{\Xi^{*}}$. For simplicity of notions, we again work on some special 3 -simplices as the model cases, whose arguments can equally be applied to all elements in $\Xi^{*}(3)$. We consider the 3 -simplices of $X_{\Xi^{*}}$ contained in the first three polytopes in (4.2), and they are $\Delta_{u}, \Delta_{d}$ of (4.2), and the eight simplices of (4.4) with $c=\mathbf{v}^{m}+\left(e^{2}+e^{4}-e^{1}-e^{3}\right) / 2(r+1)$. The affine toric coordinates for $X_{\Xi^{*}}$ are determined by the integral basis of $M$ in the simplicial cone dual to the one in $N$ generated by the corresponding 3-simplex. By computation, the affine coordinate systems corresponding to these 3 -simplices are as follows:

$$
\begin{aligned}
& \Delta_{u}:\left(V_{1}^{\left(m_{1}\right)}, V_{2}^{\left(m_{2}\right)}, V_{3}^{\left(m_{3}-1\right)}, V_{4}^{\left(m_{4}\right)}\right), \quad V_{i}^{(l)}:=\frac{Z_{i}^{r+1-l}}{\left(Z_{1} \cdots \breve{Z}_{i} \cdots Z_{4}\right)^{l}}, \\
& \Delta_{d}:\left(V_{1}^{\prime\left(m_{1}\right)}, V_{2}^{\prime\left(m_{2}+1\right)}, V_{3}^{\prime\left(m_{3}\right)}, V_{4}^{\prime\left(m_{4}\right)}\right), \quad V_{i}^{\prime(l)}:=\frac{\left(Z_{1} \cdots \breve{Z}_{i} \cdots Z_{4}\right)^{l}}{Z_{i}^{r+1-l}}, \\
& C_{i}^{c}:\left(U_{i, 1}^{(c)}, U_{i, 2}^{(c)}, U_{i, 3}^{(c)}, U_{i, 4}^{(c)}\right), \\
& U_{i, i}^{(c)}:=\frac{\left(Z_{j} Z_{j} Z_{k}\right)^{(r+1) c_{i}+1 / 2}}{Z_{i}^{(r+1)\left(1-c_{i}\right)-1 / 2}}, \quad U_{i, j}^{(c)}:=\frac{\left(Z_{i} Z_{j}\right)^{(r+1)\left(1-c_{i}-c_{j}\right)}}{Z_{k} Z_{s}^{(r+1)\left(c_{i}+c_{j}\right)}}, \\
& C_{i}^{\prime c}:\left(U_{1, i}^{\prime(c)}, U_{2, i}^{\prime(c)}, U_{3, i}^{\prime(c)}, U_{4, i}^{\prime(c)}\right), \\
& U_{i, i}^{\prime(c)}:=\frac{Z_{i}^{(r+1)\left(1-c_{i}\right)+1 / 2}}{\left(Z_{j} Z_{k} Z_{s}\right)^{(r+1) c_{i}-1 / 2}}, \quad U_{i, j}^{\prime(c)}:=\frac{\left(Z_{k} Z_{s}\right)^{(r+1)\left(1-c_{k}-c_{s}\right)}}{\left(Z_{i} Z_{j}\right)^{(r+1)\left(c_{k}+c_{s}\right)}} .
\end{aligned}
$$

Here, the indices $i, j, k, s$ indicate the four numbers by permuting $1,2,3,4$, and we will adopt this convention for the rest of this proof if no confusion will arise. Define 
the following eigenpolynomials of $G$ for $\beta \in \mathbb{C}$ and integers $l$, with $0 \leq l \leq(r+1)$ :

$$
\begin{gathered}
F_{i}^{(l)}(\beta)=Z_{i}^{l}-\beta\left(Z_{j} Z_{k} Z_{s}\right)^{(r+1)-l}, \quad G_{i, j}^{(l)}(\beta)=\left(Z_{i} Z_{j}\right)^{l}-\beta\left(Z_{k} Z_{s}\right)^{(r+1)-l}, \\
H_{i}^{(l)}(\beta)=\left(Z_{j} Z_{j} Z_{s}\right)^{l}-\beta Z_{i}^{(r+1)-l} .
\end{gathered}
$$

Let $y$ be an element of $X_{\Xi *}$. For $y$ near $x_{\Delta_{u}}$ with coordinates $\left(V_{1}^{\left(m_{1}\right)}, V_{2}^{\left(m_{2}\right)}, V_{3}^{\left(m_{3}-1\right)}\right.$, $\left.V_{4}^{\left(m_{4}\right)}\right)=\left(v_{1}, v_{2}, v_{3}, v_{4}\right)$, the ideal $I(y)$ has the generators

$$
\begin{gathered}
F_{1}^{\left(r+1-m_{1}\right)}\left(v_{1}\right), F_{2}^{\left(r+1-m_{2}\right)}\left(v_{2}\right), F_{3}^{\left(r+2-m_{3}\right)}\left(v_{3}\right), F_{4}^{\left(r+1-m_{4}\right)}\left(v_{4}\right), G_{1,2}^{\left(m_{3}+m_{4}\right)}\left(v_{1} v_{2}\right), \\
G_{1,3}^{\left(m_{2}+m_{4}+1\right)}\left(v_{1} v_{3}\right), G_{1,4}^{\left(m_{2}+m_{3}\right)}\left(v_{1} v_{4}\right), G_{2,3}^{\left(m_{1}+m_{4}+1\right)}\left(v_{2} v_{3}\right), G_{2,4}^{\left(m_{1}+m_{3}\right)}\left(v_{2} v_{4}\right), \\
G_{3,4}^{\left(m_{1}+m_{2}+1\right)}\left(v_{3} v_{4}\right), H_{1}^{\left(m_{1}+1\right)}\left(v_{2} v_{3} v_{4}\right), H_{2}^{\left(m_{2}+1\right)}\left(v_{1} v_{3} v_{4}\right) \\
H_{3}^{\left(m_{3}\right)}\left(v_{1} v_{2} v_{4}\right), H_{4}^{\left(m_{4}+1\right)}\left(v_{1} v_{2} v_{3}\right), Z_{1} Z_{2} Z_{3} Z_{4}-v_{1} v_{2} v_{3} v_{4} .
\end{gathered}
$$

For $y$ near $x_{\Delta_{d}}$ with coordinates $\left(V_{1}^{\prime\left(m_{1}\right)}, V_{2}^{\prime\left(m_{2}+1\right)}, V_{3}^{\prime\left(m_{3}\right)}, V_{4}^{\prime\left(m_{4}\right)}\right)=\left(v_{1}^{\prime}, v_{2}^{\prime}, v_{3}^{\prime}, v_{4}^{\prime}\right), I(y)$ has the generators

$$
\begin{gathered}
F_{1}^{\left(r+2-m_{1}\right)}\left(v_{2}^{\prime} v_{3}^{\prime} v_{4}^{\prime}\right), F_{2}^{\left(r+1-m_{2}\right)}\left(v_{1}^{\prime} v_{3}^{\prime} v_{4}^{\prime}\right), F_{3}^{\left(r+2-m_{3}\right)}\left(v_{1}^{\prime} v_{2}^{\prime} v_{4}^{\prime}\right), F_{4}^{\left(r+2-m_{4}\right)}\left(v_{1}^{\prime} v_{2}^{\prime} v_{3}^{\prime}\right), \\
G_{1,2}^{\left(m_{3}+m_{4}\right)}\left(v_{3}^{\prime} v_{4}^{\prime}\right), G_{1,3}^{\left(m_{2}+m_{4}+1\right)}\left(v_{2}^{\prime} v_{4}^{\prime}\right), G_{1,4}^{\left(m_{2}+m_{3}+1\right)}\left(v_{2}^{\prime} v_{3}^{\prime}\right), G_{2,3}^{\left(m_{1}+m_{4}\right)}\left(v_{1}^{\prime} v_{4}^{\prime}\right), \\
G_{2,4}^{\left(m_{1}+m_{3}\right)}\left(v_{1}^{\prime} v_{3}^{\prime}\right), G_{3,4}^{\left(m_{1}+m_{2}+1\right)}\left(v_{1}^{\prime} v_{2}^{\prime}\right), H_{1}^{\left(m_{1}\right)}\left(v_{1}^{\prime}\right), H_{2}^{\left(m_{2}+1\right)}\left(v_{2}^{\prime}\right), \\
H_{3}^{\left(m_{3}\right)}\left(v_{3}^{\prime}\right), H_{4}^{\left(m_{4}\right)}\left(v_{4}^{\prime}\right), Z_{1} Z_{2} Z_{3} Z_{4}-v_{1}^{\prime} v_{2}^{\prime} v_{3}^{\prime} v_{4}^{\prime} .
\end{gathered}
$$

For $y$ near $x_{C_{i}^{c}}$ with coordinates $\left(U_{i l}^{(c)}=u_{l}\right)_{1 \leq l \leq 4}, I(y)$ has the generators

$$
\begin{gathered}
F_{i}^{\left((r+1)\left(1-c_{i}\right)+1 / 2\right)}\left(u_{1} u_{2} u_{3} u_{4}\right), F_{j}^{\left((r+1)\left(1-c_{j}\right)+1 / 2\right)}\left(u_{i} u_{j}\right), F_{k}^{\left((r+1)\left(1-c_{k}\right)+1 / 2\right)}\left(u_{i} u_{k}\right), \\
F_{s}^{\left((r+1)\left(1-c_{s}\right)+1 / 2\right)}\left(u_{i} u_{s}\right), G_{i, j}^{(r+1)\left(c_{k}+c_{s}\right)}\left(u_{j}\right), G_{i, k}^{(r+1)\left(c_{j}+c_{s}\right)}\left(u_{k}\right), G_{i, s}^{(r+1)\left(c_{j}+c_{k}\right)}\left(u_{s}\right), \\
G_{j, k}^{(r+1)\left(c_{i}+c_{s}\right)+1}\left(u_{i}{ }^{2} u_{j} u_{k}\right), G_{j, s}^{(r+1)\left(c_{i}+c_{k}\right)+1}\left(u_{i}{ }^{2} u_{j} u_{s}\right), G_{k, s}^{(r+1)\left(c_{i}+c_{j}\right)+1}\left(u_{i}{ }^{2} u_{k} u_{s}\right), \\
H_{i}^{\left((r+1) c_{i}+1 / 2\right)}\left(u_{i}\right), H_{j}^{\left((r+1) c_{j}+1 / 2\right)}\left(u_{i} u_{k} u_{s}\right), H_{k}^{\left((r+1) c_{k}+1 / 2\right)}\left(u_{i} u_{j} u_{s}\right), \\
H_{s}^{\left((r+1) c_{s}+1 / 2\right)}\left(u_{i} u_{j} u_{k}\right), Z_{1} Z_{2} Z_{3} Z_{4}-u_{i}{ }^{2} u_{j} u_{k} u_{s} .
\end{gathered}
$$

For $y$ near $x_{C_{i}^{\prime c}}$ with coordinates $\left(U_{i l}^{\prime(c)}=u_{l}^{\prime}\right)_{1 \leq l \leq 4}, I(y)$ has the generators

$$
\begin{gathered}
F_{i}^{\left((r+1)\left(1-c_{i}\right)+1 / 2\right)}\left(u_{i}^{\prime}\right), F_{j}^{((r+1)(1-c+1 / 2)}\left(u_{i}^{\prime} u_{k}^{\prime} u_{s}^{\prime}\right), F_{k}^{\left((r+1)\left(1-c_{k}\right)+1 / 2\right)}\left(u_{i}^{\prime} u_{j}^{\prime} u_{s}^{\prime}\right), \\
F_{s}^{\left((r+1)\left(1-c_{s}\right)+1 / 2\right)}\left(u_{i}^{\prime} u_{j}^{\prime} u_{k}^{\prime}\right), G_{i, j}^{(r+1)\left(c_{k}+c_{s}\right)+1}\left(u_{i}^{\prime 2} u_{k}^{\prime} u_{s}^{\prime}\right), G_{i, k}^{(r+1)\left(c_{j}+c_{s}\right)+1}\left(u_{i}^{\prime 2} u_{j}^{\prime} u_{s}^{\prime}\right), \\
G_{i, s}^{(r+1)\left(c_{j}+c_{k}\right)+1}\left(u_{i}^{\prime 2} u_{j}^{\prime} u_{k}^{\prime}\right), G_{j, k}^{(r+1)\left(c_{i}+c_{s}\right)}\left(u_{s}^{\prime}\right), G_{j, s}^{(r+1)\left(c_{i}+c_{k}\right)}\left(u_{k}^{\prime}\right), G_{k, s}^{(r+1)\left(c_{i}+c_{j}\right)}\left(u_{j}^{\prime}\right), \\
H_{i}^{\left((r+1) c_{i}+1 / 2\right)}\left(u_{1}^{\prime} u_{2}^{\prime} u_{3}^{\prime} u_{4}^{\prime}\right), H_{j}^{\left((r+1) c_{j}+1 / 2\right)}\left(u_{i}^{\prime} u_{j}^{\prime}\right), H_{k}^{\left((r+1) c_{k}+1 / 2\right)}\left(u_{i}^{\prime} u_{k}^{\prime}\right), \\
H_{s}^{\left(c_{s}+1 / 2\right)}\left(u_{i}^{\prime} u_{s}^{\prime}\right), Z_{1} Z_{2} Z_{3} Z_{4}-u_{i}^{\prime 2} u_{j}^{\prime} u_{k}^{\prime} u_{s}^{\prime} .
\end{gathered}
$$


The centers of the above affine charts have the monomial ideals, say the one near $x_{\Delta_{u}}$, $I\left(x_{\Delta_{u}}\right)$, is obtained by setting $v_{l}=0$ in (4.7), hence a monomial ideal. There are exactly $(r+1)^{3}$ monomials not in $I\left(x_{\Delta_{u}}\right)$, that is, $\left|I\left(x_{\Delta_{u}}\right)^{\dagger}\right|=(r+1)^{3}$. For $y$ near $x_{\Delta_{u}}$, by using (4.7) and employing the Gröbner basis techniques and the toric data, one obtains the colength of $I(y)$ in $\mathbb{C}[Z]$ satisfying the relation colength $(I(y)) \leq \operatorname{colength}\left(I\left(x_{\Delta_{u}}\right)\right)=$ $(r+1)^{3}$; this implies colength $(I(y))=(r+1)^{3}$, by which an element $\lambda(y) \in \operatorname{Hilb}^{G}\left(\mathbb{C}^{4}\right)$ is determined. One can also show the colength of $I(y)$ equal to $(r+1)^{3}$ for $y$ in other affine charts using (4.8), (4.9), (4.10). The same conclusion holds for $y$ in any affine coordinate neighborhood centered at $x_{\mathfrak{k}}$, for $\varkappa \in \Xi^{*}(3)$, and one obtains an element $\lambda(y)$ in $\operatorname{Hilb}^{G}\left(\mathbb{C}^{4}\right)$, by which the morphism $\lambda: X_{\Xi *} \rightarrow \operatorname{Hilb}^{G}\left(\mathbb{C}^{4}\right)$ is defined.

Now we are going to show that $\lambda$ is an isomorphism. For $n \in \mathbb{Z}$, we denote by $\underline{n}$ the unique integer satisfying the relation

$$
n \equiv \underline{n}(\bmod r+1), \quad 0 \leq \underline{n} \leq r .
$$

We first determine the $G$-invariant monomial ideals $J_{0}$ in $\operatorname{Hilb}^{A_{r}(4)}\left(\mathbb{C}^{4}\right)$. For such a $J_{0}$, the set $J_{0}^{\dagger}:=W_{0} \backslash\left(W_{0} \cap J_{0}\right)$ forms a basis of a $G$-regular representation space. Denote by $l_{i}$ the smallest integer with $Z_{i}^{l_{i}} \in J_{0}$, by $l_{i j}$ the smallest one with $\left(Z_{i} Z_{j}\right)^{l_{i j}} \in J_{0}$ for $i \neq j$, and so on. By $1 \notin J_{0}$ and $1 \sim Z_{i}^{r+1} \sim Z_{1} Z_{2} Z_{3} Z_{4}$, we have $Z_{i}^{r+1}, Z_{1} Z_{2} Z_{3} Z_{4} \in J_{0}$, that is, $I(o) \subset J_{0}$, and the following relations hold:

$$
1 \leq l_{i j k} \leq l_{i j} \leq l_{i} \leq r+1
$$

By $J_{0}^{\perp} \subset I(o)^{\perp}$ and (2.22) for the description of the $G$-eigenspace of $I(o)^{\perp},\left(Z_{j} Z_{k} Z_{s}\right)^{r+1-l_{i}}$ is the only monomial $u \in I(o)^{\dagger}$ with $u \sim Z_{i}^{l_{i}}$, which implies $\left(Z_{j} Z_{k} Z_{s}\right)^{r+1-l_{i}} \in J_{0}^{\dagger}$ and $\left(Z_{j} Z_{k} Z_{s}\right)^{r+2-l_{i}} \in J_{0}$, hence $l_{j k s}=r+2-l_{i}$. By a similar argument, one has $l_{k s}=r+2-l_{i j}$. Hence we have

$$
l_{i}+l_{j k s}=l_{i j}+l_{k s}=r+2 \text {. }
$$

We claim that $J_{0}$ is the ideal with generators given by

$$
J_{0}=\left\langle Z_{i}^{l_{i}},\left(Z_{i} Z_{j}\right)^{l_{i j}},\left(Z_{i} Z_{j} Z_{k}\right)^{l_{i j k}}, Z_{1} Z_{2} Z_{3} Z_{4} \mid i, j, k\right\rangle .
$$

(Note that $i, j, k$ are distinct numbers among $1,2,3,4$ as before.) Let $J_{0}^{\prime}$ be the ideal in the right-hand side of (4.14). Then $I(o) \subset J_{0}^{\prime} \subset J_{0}$. Suppose $J_{0}^{\prime} \neq J_{0}$, equivalently, $J_{0} \cap J_{0}^{\prime \dagger} \neq \varnothing$. For convenience of notation but without loss of generality, we may assume $Z_{2}^{i_{2}} Z_{3}^{i_{3}} Z_{4}^{i_{4}} \in J_{0} \cap J_{0}^{\prime \dagger}$ for $i_{2} \leq i_{3} \leq i_{4}$. Hence $i_{2}<l_{234}, i_{3}<l_{34}, i_{4}<l_{4}$, which implies $p_{1}(:=$ $\left.Z_{2}^{l_{234^{-1}}} Z_{3}^{l_{34^{-1}}} Z_{4}^{l_{4}{ }^{-1}}\right) \in J_{0} \cap I(0)^{\dagger}$. By (2.22), the rest of monomials $p$ in $I(o)^{\dagger}$ with $p \sim p_{1}$ 
are given by

$$
\begin{gathered}
p_{2}:=Z \frac{r+2-l_{234}}{1} Z_{3}^{l_{34}-l_{234}} Z_{4}^{l_{4}-l_{234}}, \quad p_{3}:=Z \frac{r+2-l_{34}}{1} Z_{2}^{\frac{r+1+l_{234}-l_{34}}{2}} Z_{4}^{l_{4}-l_{34}}, \\
p_{4}:=Z_{1}^{\frac{r+2-l_{4}}{1}} Z_{2}^{\frac{r+1+l_{234}-l_{4}}{2}} Z_{3}^{\frac{r+1+l_{34}-l_{4}}{3}},
\end{gathered}
$$

among which exactly only one belongs to $J_{0}^{\dagger}$. We have $p_{1}=p_{2}$ when $l_{234}=1$. If $l_{234}>1$, by (4.13), we have $r+2-l_{234}=l_{1}$. Therefore $p_{2} \in J_{0}$. When $l_{234}=l_{34}$, we have $p_{2}=p_{3}$. When $l_{234}<l_{34}, p_{3}=\left(Z_{1} Z_{2}\right)^{l_{1,2}} Z_{2}^{l_{123}} Z_{4}^{l_{4}-l_{34}}$ by (4.13), hence $p_{3} \in J_{0}$. Similarly, $p_{3}=p_{4}$ when $l_{34}=l_{4}$. If $l_{34}<l_{4}, u_{4}=\left(Z_{1} Z_{2} Z_{3}\right)^{l_{123}} Z_{2}^{l_{234}} Z_{3}^{l_{34}}$, hence $p_{4} \in J_{0}$. Therefore $p_{i} \in J_{0}$ for $1 \leq i \leq 4$, a contradiction to their relations with $J_{0}^{\dagger}$. We are going to show that the following relations hold for $i \neq j$ :

$$
r+1 \leq l_{i}+l_{j}-l_{i j} \leq r+2 .
$$

Consider the element $w\left(:=Z_{i}^{l_{i}} Z_{j}^{l_{i j}-1} Z_{k}^{l_{i j k}-1}\right)$ in $J_{0}$. Among the monomials

$$
\begin{gathered}
w_{1}=Z_{i}^{l_{i}-l_{i j k}+1} Z_{j}^{l_{i j}-l_{i j k}} Z_{\bar{s}}^{r+2-l_{i j k}}, \quad w_{2}=Z_{i}^{l_{i}-l_{i j}+1} Z_{k}^{r+1-l_{i j}+l_{i j k}} \\
w_{3}=Z_{j}^{r-l_{i}+l_{i j}} Z_{k}^{r-l_{i}+l_{i j k}} Z_{s}^{r+1-l_{i}}
\end{gathered}
$$

$G$-equivalent to $w$, there exists exactly one in $J_{0}^{\dagger}$. It is easy to see that $w_{1}=$ $Z_{i}^{l_{i}-l_{i j k}+1} Z_{j}^{l_{i j}-l_{i j k}} Z_{s}^{l_{s}} \in J_{0}$ unless $l_{i j k}=1$, in which case $w_{1}=w \in J_{0}$ if $l_{i}<r+1$, and $w_{1}=$ $w_{3}$ if $l_{i}=r+1$. We have $w_{1}=w_{2}$ if $l_{i j}=l_{i j k}$. When $l_{i j}>l_{i j k}, w_{2}=Z_{i}^{l_{i}-l_{i j}+1} Z_{k}^{l_{k s}+l_{i j k}-1} Z_{s}^{l_{k s}}$ $\in J_{0}$. Therefore $w_{3}$ is the element of $J_{0}^{\dagger} G$-equivalent to $w$, which, by the expression of the power of $Z_{j}$, implies

$$
r+1 \leq l_{i}+l_{j}-l_{i j}
$$

As a consequence of the above inequality, we have $l_{j}=r+1$ and $l_{i}+l_{j}-l_{i j}=r+1$ when $l_{i j}=l_{i}$; in particular, (4.16) holds. Hence we may assume $l_{i j}<l_{i}$, in which case $h:=Z_{i}^{l_{i}-1} Z_{j}^{l_{i j}} Z_{k}^{l_{i j k}-1} \in J_{0}$. Among the monomials

$$
\begin{gathered}
h_{1}=Z_{i}^{l_{i}-l_{i j k}} Z_{j}^{l_{i j}-l_{i j k}+1} Z_{s}^{r+2-l_{i j k}}, \quad h_{2}=Z_{i}^{l_{i}-l_{i j}-1} Z_{k}^{r-l_{i}+l_{i j k}} Z_{s}^{r+1-l_{i j}}, \\
h_{3}=Z_{j}^{r+2-l_{i}+l_{i j}} Z_{k}^{r+1-l_{j}+l_{i j k}} Z_{s}^{r+2-l_{i}}
\end{gathered}
$$

$G$-equivalent to $h$, there exists exactly one in $J_{0}^{\dagger}$. We have $h_{1}=h \in J_{0}$ if $l_{i j k}=1$. When $l_{i j k}>1, h_{1}=Z_{i}^{l_{i}-l_{i j k}} Z_{j}^{l_{i j}-l_{i j k}+1} Z_{s}^{l_{s}}$ and $h_{1} \in J_{0}$. One has $h_{3}=Z_{j}^{l_{i j}} Z_{k}^{l_{i j k}-1}\left(Z_{j} Z_{k} Z_{s}\right)^{l_{j k s}} \in J_{0}$ unless $l_{i}=l_{i j}+1$, in which case $h_{3}=h_{2}$. Therefore we have $h_{2} \in J_{0}^{\dagger}$, which implies $l_{i}-l_{i j}-1 \leq l_{i k s}-1$, hence $l_{i}+l_{j}-l_{i j} \leq r+2$ by (4.13). Therefore we obtain relation (4.16). With $(i, j)=(1,2),(3,4)$ in (4.16), (4.13), we have $3 r+4 \leq \sum_{j=1}^{4} l_{j} \leq 3 r+6$. Using (4.13), one obtains all the possible cases of $l_{i}+l_{j}-l_{i j}$ for a given value of $\sum_{j=1}^{4} l_{j}$; consequently, all the values of $l_{I}$ 's are determined by $l_{i}$ 's. By comparing the polynomials at the origin 
in (4.7), (4.8), (4.9), (4.10), $J_{0}=I\left(x_{\mathfrak{\varkappa}}\right)$ for $\varkappa \in \Xi^{*}$ (3) by the following relations:

$$
\begin{gathered}
\Delta_{u}: \sum_{j=1}^{4} l_{j}=3 r+4, \quad l_{i j}=l_{i}+l_{j}-r-1, \\
\Delta_{d}: \sum_{j=1}^{4} l_{j}=3 r+6, \quad l_{i j}=l_{i}+l_{j}-r-2, \\
C_{i}^{c}: \sum_{j=1}^{4} l_{j}=3 r+5, \quad l_{i j}=l_{i}+l_{j}-r-2, l_{k s}=l_{k}+l_{s}-r-1, \\
C_{i}^{\prime c}: \sum_{j=1}^{4} l_{j}=3 r+5, \quad l_{i j}=l_{i}+l_{j}-r-1, l_{k s}=l_{k}+l_{s}-r-2,
\end{gathered}
$$

where the indices in toric data are connected to the $l_{i}$ 's by the following relations:

$$
\begin{gathered}
\Delta_{u}: l_{3}=r+2-m_{3}, \quad l_{j}=r+1-m_{j} \quad(j \neq 3), \\
\Delta_{d}: l_{2}=r+1-m_{2}, \quad l_{j}=r+2-m_{j} \quad(j \neq 2), \\
C_{i}^{c}, C_{i}^{\prime c}: l_{j}=(r+1)\left(1-c_{j}\right)+\frac{1}{2}, \quad c=\frac{1}{2 r+2} \sum_{j=1}^{4}\left(2 r+3-2 l_{j}\right) e^{j} .
\end{gathered}
$$

With $l_{i}^{\prime}:=r+1-l_{i}$, the $l_{i}^{\prime \prime}$ s are 4 positive integers satisfying the equation $\sum_{i=1}^{4} l_{i}^{\prime}=L^{\prime}$ with $L^{\prime}=r, r-1, r-2$. The number of solutions of $l_{i}^{\prime}$ 's is equal to $\left(L_{3}^{\prime}+3\right)$. Hence one obtains the following numbers of $\varkappa \in \Xi^{*}$ (3) for the toric data in (4.2), (4.4) using the relation with $l_{i}$ 's:

$$
\begin{gathered}
\#\left\{\Delta_{u}\right\}=\frac{(r+1)(r+2)(r+3)}{6}, \quad \#\left\{\Delta_{d}\right\}=\frac{(r-1) r(r+1)}{6}, \\
\#\{c\}=\frac{r(r+1)(r+2)}{6} .
\end{gathered}
$$

Let $J$ be a $G$-invariant ideal representing an element in $\operatorname{Hilb}^{G}\left(\mathbb{C}^{4}\right)$. With the Gröbner basis argument as in Theorem 3.4, there is a monomial ideal $J_{0}$ in $\operatorname{Hilb}^{G}\left(\mathbb{C}^{4}\right)$ such that $J_{0}^{\dagger}$ gives rise to a basis of $\mathbb{C}[Z] / J$ with relation (3.15). As $J_{0}=I\left(x_{\mathfrak{k}}\right)$ for some $\mathfrak{R} \in$ $\Xi^{*}(3)$, which is determined by the integers $l_{i}, l_{i j}, l_{i j k}$ with the relations in (4.13), (4.20), this implies that for some $\gamma_{i}, \gamma_{i j}, \gamma_{j k s}, \gamma_{1234} \in \mathbb{C}$, the polynomials $F_{i}^{\left(l_{i}\right)}\left(\gamma_{i}\right), G_{i j}^{\left(l_{i j}\right)}\left(\gamma_{i j}\right)$, $H_{i}^{\left(l_{j k s}\right)}\left(\gamma_{j k s}\right)$, and $Z_{1} Z_{2} Z_{3} Z_{4}-\gamma_{1234}$ are elements of $J$. From the expressions of $F_{i}^{(l)}(\beta)$, $G_{i, j}^{(l)}(\beta), H_{i}^{(l)}(\beta)$, and using $\operatorname{dim}(\mathbb{C}[Z] / J)=(r+1)^{3}$, one can conclude that

$$
J=\left\langle F_{i}^{\left(l_{i}\right)}\left(\gamma_{i}\right), G_{i j}^{\left(l_{i j}\right)}\left(\gamma_{i j}\right), H_{i}^{\left(l_{j k s}\right)}\left(\gamma_{j k s}\right), Z_{1} Z_{2} Z_{3} Z_{4}-\gamma_{1234}\right\rangle_{i, j, k, s} .
$$

We are going to determine the relations among the $\gamma_{I}$ 's using the relations (4.13), (4.20) and according to the type of $l_{i}$ 's. By

$$
\left(\gamma_{1234}-\gamma_{123} \gamma_{4}\right) Z_{4}^{l_{4}-1}=Z_{1} Z_{2} Z_{3} F_{4}^{\left(l_{4}\right)}\left(\gamma_{4}\right)-\gamma_{4} H_{4}^{\left(l_{123}\right)}\left(\gamma_{123}\right)-Z_{4}^{l_{4}-1}\left(Z_{1} Z_{2} Z_{3} Z_{4}-\gamma_{1234}\right) \in J
$$

and $Z_{4}^{l_{4}-1} \notin J$, we have $\gamma_{1234}=\gamma_{123} \gamma_{4}$. 
For $J$ with $J_{0}$ of type $\Delta_{u}$, by (4.20), we have

$$
\begin{aligned}
& \left(\gamma_{123}-\gamma_{12} \gamma_{3}\right) Z_{3}^{l_{34}-1} Z_{4}^{l_{4}-1} \\
& \quad=\left(Z_{1} Z_{2}\right)^{l_{123}} F_{3}^{\left(l_{3}\right)}\left(\gamma_{3}\right)+\gamma_{3} Z_{4}^{l_{124}-1} G_{12}^{\left(l_{12}\right)}\left(\gamma_{12}\right)-Z_{3}^{l_{34}-1} H_{4}^{\left(l_{123}\right)}\left(\gamma_{123}\right), \\
& \left(\gamma_{13}-\gamma_{1} \gamma_{3}\right) Z_{3}^{l_{234}-1}\left(Z_{2} Z_{4}\right)^{l_{24}-1} \\
& \quad=\gamma_{3}\left(Z_{2} Z_{4}\right)^{l_{124}-1} F_{1}^{\left(l_{1}\right)}\left(\gamma_{1}\right)+Z_{1}^{l_{13}} F_{3}^{\left(l_{3}\right)}\left(\gamma_{3}\right)-Z_{3}^{l_{3}-l_{13}} G_{13}^{\left(l_{13}\right)}\left(\gamma_{13}\right),
\end{aligned}
$$

which are elements in $J$. By $Z_{3}^{l_{34}-1} Z_{4}^{l_{4}-1}, Z_{3}^{l_{234}-1}\left(Z_{2} Z_{4}\right)^{l_{24}-1} \in J_{0}^{\dagger}$, we have $\gamma_{123}=\gamma_{1} \gamma_{23}$, $\gamma_{23}=\gamma_{2} \gamma_{3}$. By permuting the indices, one obtains $\gamma_{I}=\prod_{i \in I} \gamma_{i}$ for a subset $I$ of $\{1,2,3,4\}$. By (4.7), (4.22), we have $J=I(y)$ for $y$ near $x_{\Delta_{u}}$ with the coordinates $v_{i}=\gamma_{i}$.

When $J_{0}$ is of type $\Delta_{u}$, by (4.20), the following elements are in $J$ :

$$
\begin{aligned}
& \left(\gamma_{12} \gamma_{134}-\gamma_{1}\right) Z_{2}^{l_{234}-1}\left(Z_{3} Z_{4}\right)^{l_{34}-1} \\
& \quad=\left(Z_{3} Z_{4}\right)^{l_{134}} F_{1}^{\left(l_{1}\right)}\left(\gamma_{1}\right)-\gamma_{134} Z_{2}^{l_{234}-1} G_{12}^{\left(l_{12}\right)}\left(\gamma_{12}\right)-Z_{1}^{l_{12}} H_{2}^{\left(l_{134}\right)}\left(\gamma_{134}\right), \\
& \left(\gamma_{12}-\gamma_{123} \gamma_{124}\right) Z_{3}^{l_{3}-1} Z_{4}^{l_{34}-1} \\
& \quad=-Z_{3}^{l_{123}} G_{12}^{\left(l_{12}\right)}\left(\gamma_{12}\right)+\gamma_{123} Z_{4}^{l_{34}-1} H_{3}^{\left(l_{124}\right)}\left(\gamma_{124}\right)+\left(Z_{1} Z_{2}\right)^{l_{124}} H_{4}^{\left(l_{123}\right)}\left(\gamma_{123}\right) .
\end{aligned}
$$

Therefore $\gamma_{1}=\gamma_{12} \gamma_{134}$ and $\gamma_{12}=\gamma_{123} \gamma_{124}$. Set $v_{i}^{\prime}=\gamma_{1 \cdots{ }^{\prime} \cdots 4}$. With the same argument, one obtains $\gamma_{I}=\prod_{j \in I^{\prime}} v_{j}^{\prime}$ for $I \neq 1234$, where $I^{\prime}$ is the complement set of $I$ in $\{1,2,3,4\}$. Therefore, by (4.8), (4.22), $J=I(y)$ for $y$ near $x_{\Delta_{d}}$ having $v_{i}^{\prime}$ 's as coordinates.

When $J_{0}$ is of type $C_{i}^{c}$ or $C_{i}^{\prime c}$, without loss of generality, we may assume $i=1$. In the case $C_{1}^{c}$, the following elements are in $J$, by (4.20):

$$
\begin{aligned}
& \left(\gamma_{123}-\gamma_{13} \gamma_{2}\right)\left(Z_{1} Z_{3}\right)^{l_{134}-1} Z_{4}^{l_{4}-1} \\
& \quad=\gamma_{13} Z_{4}^{l_{24}-1} F_{2}^{\left(l_{2}\right)}\left(\gamma_{2}\right)+Z_{2}^{l_{13}-l_{134}+1} G_{13}^{\left(l_{13}\right)}\left(\gamma_{13}\right)-\left(Z_{1} Z_{3}\right)^{l_{134}-1} H_{4}^{\left(l_{123}\right)}\left(\gamma_{123}\right), \\
& \left(\gamma_{2}-\gamma_{12} \gamma_{234}\right) Z_{1}^{l_{134}-1}\left(Z_{3} Z_{4}\right)^{l_{34}-1} \\
& \quad=-\left(Z_{3} Z_{4}\right)^{l_{234}} F_{2}^{\left(l_{2}\right)}\left(\gamma_{2}\right)+\gamma_{234} Z_{1}^{l_{134}-1} G_{12}^{\left(l_{12}\right)}\left(\gamma_{12}\right)+Z_{2}^{l_{12}} H_{1}^{\left(l_{234}\right)}\left(\gamma_{234}\right), \\
& \left(\gamma_{1}-\gamma_{12} \gamma_{134}\right) Z_{2}^{l_{234}-1}\left(Z_{3} Z_{4}\right)^{l_{34}-1} \\
& \quad=-\left(Z_{3} Z_{4}\right)^{l_{1}-l_{1,2}} F_{1}^{\left(l_{1}\right)}\left(\gamma_{1}\right)+\gamma_{134} Z_{2}^{l_{234}-1} G_{12}^{\left(l_{12}\right)}\left(\gamma_{1,2}\right)+Z_{1}^{l_{12}} H_{2}^{\left(l_{134}\right)}\left(\gamma_{134}\right), \\
& \left(\gamma_{23}-\gamma_{2} \gamma_{3}\right)\left(Z_{2}\right)^{l_{124}-1}\left(Z_{1} Z_{4}\right)^{l_{14}-1} \\
& \quad=\gamma_{2}\left(Z_{1} Z_{4}\right)^{l_{3}-l_{23}} F_{3}^{\left(l_{3}\right)}\left(\gamma_{3}\right)+Z_{3}^{l_{23}} F_{2}^{\left(l_{2}\right)}\left(\gamma_{2}\right)-Z_{2}^{l_{124}-1} G_{23}^{\left(l_{23}\right)}\left(\gamma_{23}\right) .
\end{aligned}
$$


Hence

$$
\gamma_{123}=\gamma_{2} \gamma_{13}, \quad \gamma_{2}=\gamma_{234} \gamma_{12}, \quad \gamma_{1}=\gamma_{12} \gamma_{134}, \quad \gamma_{23}=\gamma_{2} \gamma_{3},
$$

which are the same relations as $u_{I}$ 's in (4.9) for $i=1$ under the identification $u_{1}=\gamma_{234}$ and $u_{j}=\gamma_{1 j}$ for $j \neq 1$. By permuting the indices, one can show that all the remaining relations in (4.9) are satisfied in terms of the $\gamma_{I}$ 's. Hence, by (4.22), $J=I(y)$ for $y$ near $x_{C_{1}}$ with the $u_{i}$ 's as the coordinates of $y$.

For $J_{0}$ of type $C_{1}^{\prime c}$, the following elements are in $J$, by (4.20):

$$
\begin{aligned}
& \left(\gamma_{234}-\gamma_{34} \gamma_{2}\right) Z_{1}^{l_{1}-1} Z_{2}^{l_{12}-1} \\
& \quad=\left(Z_{3} Z_{4}\right)^{l_{234}} F_{2}^{\left(l_{2}\right)}\left(\gamma_{2}\right)+\gamma_{2} Z_{1}^{l_{1}-l_{12}} G_{34}^{\left(l_{34}\right)}\left(\gamma_{34}\right)-Z_{2}^{l_{12}-1} H_{1}^{\left(l_{234}\right)}\left(\gamma_{234}\right), \\
& \left(\gamma_{2}-\gamma_{23} \gamma_{124}\right)\left(Z_{1} Z_{4}\right)^{l_{134}-1} Z_{3}^{l_{3}-1} \\
& \quad=-Z_{3}^{l_{23}} F_{2}^{\left(l_{2}\right)}\left(\gamma_{2}\right)+Z_{2}^{l_{124}} G_{23}^{l_{2,3}}\left(\gamma_{23}\right)+\gamma_{23}\left(Z_{1} Z_{4}\right)^{l_{134}-1} H_{3}^{\left(l_{124}\right)}\left(\gamma_{124}\right), \\
& \left(\gamma_{124}-\gamma_{1} \gamma_{24}\right) Z_{1}^{l_{13}-1} Z_{3}^{l_{3}-1} \\
& \quad=\left(Z_{2} Z_{4}\right)^{l_{124}} F_{1}^{\left(l_{1}\right)}\left(\gamma_{1}\right)+\gamma_{1} Z_{3}^{l_{3}-l_{1,3}} G_{24}^{\left(l_{24}\right)}\left(\gamma_{24}\right)-Z_{1}^{l_{1,3}-1} H_{3}^{\left(l_{124}\right)}\left(\gamma_{124}\right), \\
& \left(\gamma_{12}-\gamma_{1} \gamma_{2}\right) Z_{2}^{l_{234}-1}\left(Z_{3} Z_{4}\right)^{l_{34}-1} \\
& \quad=\gamma_{2}\left(Z_{3} Z_{4}\right)^{l_{1}-l_{12}} F_{1}^{\left(l_{1}\right)}\left(\gamma_{1}\right)+Z_{1}^{l_{12}} F_{2}^{\left(l_{2}\right)}\left(\gamma_{2}\right)-Z_{2}^{l_{234}-1} G_{12}^{l_{12}}\left(\gamma_{12}\right) .
\end{aligned}
$$

Hence

$$
\gamma_{234}=\gamma_{34} \gamma_{2}, \quad \gamma_{2}=\gamma_{23} \gamma_{124}, \quad \gamma_{124}=\gamma_{1} \gamma_{24}, \quad \gamma_{12}=\gamma_{1} \gamma_{2},
$$

which are the same relations of $u_{I}^{\prime}$ 's in (4.10) for $i=1$ under the identifications $u_{1}^{\prime}=\gamma_{1}$, $u_{2}^{\prime}=\gamma_{34}, u_{3}^{\prime}=\gamma_{24}, u_{4}^{\prime}=\gamma_{23}$. By a similar argument, all the relations of (4.10) hold; therefore $J=I(y)$ for $y$ near $x_{C_{1}^{\prime}}$ having the coordinates $u_{i}^{\prime}$ 's.

By the results we have obtained, one concludes that $\operatorname{Hilb}^{G}\left(\mathbb{C}^{4}\right)$ is a smooth toric variety, hence of the form $X_{\Xi^{* *}}$, where $\Xi^{* *}$ is a simplicial decomposition of $\Delta$ which is a refinement of $\Xi^{*}$ corresponding to the morphism $\lambda$. Indeed, the above analysis of local structure of $\operatorname{Hilb}^{G}\left(\mathbb{C}^{4}\right)$ has shown that $\Xi^{*}=\Xi^{* *}$, therefore $\lambda$ is an isomorphism between $X_{\Xi^{*}}$ and $\mathrm{Hilb}^{G}\left(\mathbb{C}^{4}\right)$. The number of exceptional divisors appearing in the canonical bundle of $X_{\Xi *}$ is equal to $r(r+1)(r+2) / 6$ by $(4.22)$.

5. $G$-Hilbert scheme over $\mathbb{C}^{3} / \mathfrak{A}_{4}$. It is known that the alternating group $\mathfrak{A}_{n+1}$ is a simple group except when $n=2,3$, in which cases $\mathfrak{A}_{3} \simeq \mathbb{Z}_{3}$ and $\mathfrak{A}_{4}$ is isomorphic to the ternary trihedral group $\left(\mathbb{Z}_{2} \times \mathbb{Z}_{2}\right) \triangleleft \mathbb{Z}_{3}$. The $G$-Hilbert scheme for $\mathbb{A}_{3}$ is the minimal resolution of $\mathbb{C}^{2} / \mathfrak{A}_{3}$. In this section, we are going to give a constructive proof of the smooth and explicit crepant structure of $\operatorname{Hilb}^{\mathfrak{A}_{4}}\left(\mathbb{C}^{3}\right)$.

THEOREM 5.1. Hilb $^{\mathfrak{A}_{4}}\left(\mathbb{C}^{3}\right)$ is a smooth variety with a trivial canonical bundle. 
We will devote the rest of this section to the proof of the above theorem and always denote $G=\mathfrak{A}_{4}$. Introduce the following coordinates $\left(z_{1}, z_{2}, z_{3}\right)$ of $V$ in $(2.23)_{n=3}$ :

$$
z_{1}=-\tilde{z}_{1}+\tilde{z}_{2}+\tilde{z}_{3}-\tilde{z}_{4}, \quad z_{2}=\tilde{z}_{1}-\tilde{z}_{2}+\tilde{z}_{3}-\tilde{z}_{4}, \quad z_{3}=\tilde{z}_{1}+\tilde{z}_{2}-\tilde{z}_{3}-\tilde{z}_{4},
$$

where $\sum_{j=1}^{4} \bar{z}_{j}=0$. The irreducible representation of $G$ on $\mathbb{C}^{3}(=V)$, denoted by 3 , has the following matrix forms for generators of $G$ :

$$
\begin{aligned}
(12)(34) \longmapsto\left(\begin{array}{ccc}
-1 & 0 & 0 \\
0 & -1 & 0 \\
0 & 0 & 1
\end{array}\right), \quad(13)(24) \longmapsto\left(\begin{array}{ccc}
-1 & 0 & 0 \\
0 & 1 & 0 \\
0 & 0 & -1
\end{array}\right), \\
(123) \longmapsto\left(\begin{array}{lll}
0 & 1 & 0 \\
0 & 0 & 1 \\
1 & 0 & 0
\end{array}\right) .
\end{aligned}
$$

There are 4 distinct irreducible $G$-modules, $\operatorname{Irr}(G)=\left\{\mathbf{1}, \mathbf{1}_{\omega}, \mathbf{1}_{\bar{\omega}}, \mathbf{3}\right\}$, where $\omega:=e^{2 \pi \sqrt{-1} / 3}$, and $\mathbf{1}_{*}$ is the $G$-character determined only by the (123)-value $*$. Using the coordinates $\left(z_{i}\right)_{i=1}^{3}$ of $\mathbb{C}^{3}$, the generators of $G$-invariant polynomials in $\mathbb{C}[Z]$ are

$$
\begin{gathered}
Y_{1}=Z_{1}^{2}+Z_{2}^{2}+Z_{3}^{2}, \quad Y_{2}=Z_{1} Z_{2} Z_{3}, \\
Y_{3}=Z_{1}^{2} Z_{2}^{2}+Z_{2}^{2} Z_{3}^{2}+Z_{3}^{2} Z_{1}^{2}, \quad X=\left(Z_{1}^{2}-Z_{2}^{2}\right)\left(Z_{2}^{2}-Z_{3}^{2}\right)\left(Z_{3}^{2}-Z_{1}^{2}\right) .
\end{gathered}
$$

Note that the above variables are related to $s_{2}, s_{3}, s_{4}, d$ in $(2.25)_{n=3}$ by the relations $Y_{1}=-8 s_{2}, Y_{2}=-8 s_{3}, Y_{3}=16 s_{2}^{2}-64 s_{4}, X=64 d$. The $G$-invariant polynomial relation (2.25) with $F_{3}$ in (2.26) becomes

$$
X^{2}=-4 Y_{1}^{3} Y_{2}^{2}-27 Y_{2}^{4}+18 Y_{1} Y_{2}^{2} Y_{3}+Y_{1}^{2} Y_{3}^{2}-4 Y_{3}^{3} .
$$

Let $\mathbb{C}[Z]_{j}$ be the space of homogeneous polynomials of degree $j$, and denote $I(o)_{j}^{\perp}=$ $I(o)^{\perp} \cap \mathbb{C}[Z]_{j}$. Then $I(o)_{j}^{\perp}$ is a $G$-submodule of $I(o)^{\perp}$. In fact, the only nonzero $I(o)_{j}^{\perp}$ 's are in the range $0 \leq j \leq 5$, whose $G$-irreducible factors are as follows (for an equivalent form, see, e.g., [6, Table 2.2]):

$$
\begin{array}{rlrl}
I(o)_{0}^{\perp} & =m_{0} \simeq \mathbf{1}, & & m_{0}=\mathbb{C}, \\
I(o)_{1}^{\perp} & =m_{1} \simeq \mathbf{3}, & & m_{1}=\left\{Z_{1}, Z_{2}, Z_{3}\right\}, \\
I(o)_{\frac{\perp}{2}} & =m_{2}+m_{3}+m_{4} & & m_{2}=\{f\}, m_{3}=\{\bar{f}\}, \\
& \simeq \mathbf{1}_{\bar{\omega}}+\mathbf{1}_{\omega}+3, & & m_{4}=\left\{Z_{2} Z_{3}, Z_{3} Z_{1}, Z_{1} Z_{2}\right\}, \\
I(o)_{\frac{1}{3}} & =m_{5}+m_{6} \simeq \mathbf{3}+\mathbf{3}, & & m_{5}=f\left\{Z_{1}, \omega^{2} Z_{2}, \omega Z_{3}\right\}, m_{6}=\bar{f}\left\{Z_{1}, \omega Z_{2}, \omega^{2} Z_{3}\right\}, \\
I(o)_{4}^{\perp} & =m_{7}+m_{8}+m_{9} & & m_{7}=\left\{\bar{f}^{2}\right\}, m_{8}=\left\{f^{2}\right\}, \\
& \simeq \mathbf{1}_{\bar{\omega}}+\mathbf{1}_{\omega}+3, & & m_{9}=f\left\{\omega Z_{1} Z_{2}, Z_{2} Z_{3}, \omega^{2} Z_{3} Z_{1}\right\}, \\
I(o)_{5}^{\perp} & =m_{10} \simeq 3, & m_{10}=\bar{f}^{2}\left\{Z_{1}, \omega^{2} Z_{2}, \omega Z_{3}\right\},
\end{array}
$$


TABLE 5.1

\begin{tabular}{|l|l|}
\hline$J_{0}$ & $\mathbb{C}[Z] / J_{0}$ \\
\hline$x_{0}:=\langle f\rangle+I(o)$ & $m_{0}+m_{1}+m_{3}+m_{4}+m_{6}+m_{7}$ \\
$x_{0}^{\prime}:=\langle\bar{f}\rangle+I(o)$ & $m_{0}+m_{1}+m_{2}+m_{4}+m_{5}+m_{8}$ \\
$x_{\infty}:=\left\langle Z_{1} f, \omega^{2} Z_{2} f, \omega Z_{3} f, \bar{f}^{2}\right\rangle+I(o)$ & $m_{0}+m_{1}+m_{2}+m_{3}+m_{4}+m_{6}$ \\
$x_{\infty}^{\prime}:=\left\langle Z_{1} \bar{f}, \omega Z_{2} \bar{f}, \omega^{2} Z_{3} \bar{f}, f^{2}\right\rangle+I(o)$ & $m_{0}+m_{1}+m_{2}+m_{3}+m_{4}+m_{5}$ \\
\hline
\end{tabular}

where $f:=\sum_{j=1}^{3} \omega^{j-1} Z_{j}^{2}, \bar{f}:=\sum_{j=1}^{3} \omega^{2 j-2} Z_{j}^{2}$. We have the $G$-irreducible decomposition $I(o)^{\perp}=\sum_{k=0}^{10} m_{k}$. Note that $f \bar{f}, f^{3}, \bar{f}^{3}$ are $G$-invariant polynomials with the following relations:

$$
f \bar{f}=Y_{1}^{2}-3 Y_{3}, \quad f^{3}-\bar{f}^{3}=3\left(\omega^{2}-\omega\right) X, \quad f^{3}+\bar{f}^{3}=27 Y_{2}^{2}-9 Y_{1} Y_{3}+2 Y_{1}^{3} .
$$

LEMMA 5.2. Among $m_{k}$ 's $(1 \leq k \leq 10)$, the following tree diagram holds:

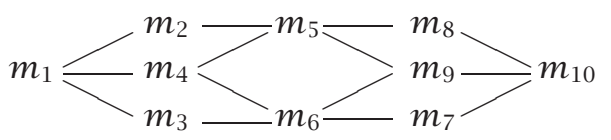

where the $m_{j}$ of the right end of an edge is contained in the ideal generated by the $m_{i}$ of the left end of the edge and $I(o)$.

Proof. By the expression of $m_{k}$, all the relations in the above diagram are trivial except the following ones:

$$
m_{9} \subset m_{6}+I(o), \quad m_{10} \subset m_{8}+I(o), \quad m_{10} \subset m_{9}+I(o) .
$$

Define the irreducible $G$-submodules of $\mathbb{C}[Z]$, isomorphic to 3 , as $\bar{m}_{9}=\bar{f}\left\{\omega^{2} Z_{1} Z_{2}\right.$, $\left.Z_{2} Z_{3}, \omega Z_{3} Z_{1}\right\}, \bar{m}_{10}:=f^{2}\left\{Z_{1}, \omega Z_{2}, \omega^{2} Z_{3}\right\}$. Then we have the equalities of ideals in $\mathbb{C}[Z]$, $\left\langle m_{9}, I(o)\right\rangle=\left\langle\bar{m}_{9}, I(o)\right\rangle,\left\langle m_{10}, I(o)\right\rangle=\left\langle\bar{m}_{10}, I(o)\right\rangle$, which imply the relations in (5.8).

We will call an ideal $J_{0}$ in $\operatorname{Hilb}^{G}\left(\mathbb{C}^{3}\right)$ central if $J_{0}$ is generated by $I(o)$ and a finite number of $m_{k}$ 's. (The central ideal $J_{0}$ here will play a role similar to that of monomial ideals in previous sections for the case of abelian groups $G$.) By Lemma 5.2, there are exactly 4 central ideals $J_{0}$ with the $G$-irreducible decomposition of $\mathbb{C}[Z] / J_{0}$ presented in Table 5.1. Note that the $J_{0}$ 's in Table 5.1 are characterized as the ideals in $\operatorname{Hilb}^{G}\left(\mathbb{C}^{3}\right)$ with monomial polynomial generators in $\mathbb{C}[Z]$. All the above four elements lie over $o \in S_{G}$ under the morphism $\sigma_{\text {Hilb }}$ of (2.9). By the analysis in [6, Section 2.5], $\sigma_{\mathrm{Hilb}}^{-1}(o)$ consists of a tree of three smooth rational curves $L+l+L^{\prime}$. Here are the locations of $J_{0}$ 's in $\sigma_{\text {Hilb }}^{-1}(o): x_{0} \in(L \backslash l) \cup L^{\prime}, x_{\infty}=L \cap l, x_{\infty}^{\prime}=L^{\prime} \cap l, x_{0}^{\prime} \in\left(L^{\prime} \backslash l\right) \cup L$ (see Figure 5.1). We are going to show that every $J$ in $\operatorname{Hilb}^{G}\left(\mathbb{C}^{3}\right)$ can be deformed to one $J_{0}$ in Table 5.1. For $J \in \operatorname{Hilb}^{G}\left(\mathbb{C}^{3}\right)$, denote by $h(J)$ the homogenous ideal in $\mathbb{C}[Z]$ generated by the highest-total-degree part of elements in $J$. As the top degree of a polynomial in $\mathbb{C}[Z]$ is preserved under the $G$-action, $h(J)$ is $G$-invariant. By applying the Gröbner basis technique with a monomial order of total degree in $\mathbb{C}[Z]$, one obtains the same ideal, $\operatorname{lt}(J)=\operatorname{lt}(h(J))$, hence a set of monomial elements in $\mathbb{C}[Z]$ which represent the basis for 


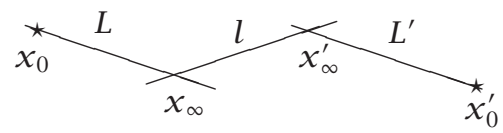

FIGURE 5.1. Tree configuration of $\sigma_{\text {Hilb }}^{-1}(o)$ for $G=\mathfrak{A}_{4}$.

both $\mathbb{C}[Z] / J$ and $\mathbb{C}[Z] / h(J)$. Therefore $h(J)$ is a homogeneous ideal in Hilb ${ }^{G}\left(\mathbb{C}^{3}\right)$. Note that $\sigma_{\text {Hilb }}(h(J))=o$. By [6, (2.4)], $h(J) \in\left\{x_{0}, x_{0}^{\prime}\right\} \cup l$. Hence $h(J)$ and $J$ can be deformed to an element in Table 5.1. Now we are going to determine the local structure near these four central elements in $\operatorname{Hilb}^{G}\left(\mathbb{C}^{3}\right)$.

For $J$ near the element $x_{\infty}$ in $\operatorname{Hilb}^{G}\left(\mathbb{C}^{3}\right)$, we have

$$
J=\left\langle\bar{f}^{2}-v_{0} f, m_{5}-v_{1} m_{6}-v_{2} m_{4}-v_{3} m_{1}, Y_{1}-\eta_{1}, Y_{2}-\eta_{2}, Y_{3}-\eta_{3}, X-\xi\right\rangle,
$$

where $\left(\xi, \eta_{1}, \eta_{2}, \eta_{3}\right)$ satisfies (5.4) and $m_{5}-v_{1} m_{6}-v_{2} m_{4}-v_{3} m_{1}$ is the $G$-module $\sum_{j=1}^{3} \mathbb{C} p_{j}$ with $p_{1}:=f Z_{1}-v_{1} \bar{f} Z_{1}-v_{2} Z_{2} Z_{3}-v_{3} Z_{1}, p_{2}:=f \omega^{2} Z_{2}-v_{1} \bar{f} \omega Z_{2}-v_{2} Z_{3} Z_{1}-$ $v_{3} Z_{2}, p_{3}:=f \omega Z_{3}-v_{1} \bar{f} \omega^{2} Z_{3}-v_{2} Z_{1} Z_{2}-v_{3} Z_{3}$. By

$$
\begin{gathered}
f^{2}-\left(v_{1} \eta_{1}+v_{3}\right) \bar{f}=Z_{1} p_{1}+\omega^{2} Z_{2} p_{2}+\omega Z_{3} p_{3}+v_{1}\left(Y_{1}-\eta_{1}\right) \bar{f} \in J, \\
\left(\eta_{1}-v_{3}-v_{0} v_{1}\right) f=Z_{1} p_{1}+\omega Z_{2} p_{2}+\omega^{2} Z_{3} p_{3}+v_{1}\left(\bar{f}^{2}-v_{0} f\right)-\left(Y_{1}-\eta_{1}\right) f \in J,
\end{gathered}
$$

and the first relation of (5.6), we have $\left(3 \eta_{3}-\eta_{1}^{2}-v_{0}\left(v_{3}+v_{1} \eta_{1}\right)\right) f \in J$. As $f \notin J$, we have

$$
\eta_{1}-v_{3}-v_{0} v_{1}=0, \quad 3 \eta_{3}-\eta_{1}^{2}=v_{0}\left(v_{3}+v_{1} \eta_{1}\right)
$$

By the relations $3 Y_{2} f-v_{2}\left(Z_{2}^{2} Z_{3}^{2}+\omega Z_{3}^{2} Z_{1}^{2}+\omega^{2} Z_{1}^{2} Z_{2}^{2}\right)=Z_{2} Z_{3} p_{1}+\omega Z_{3} Z_{1} p_{2}+\omega^{2} Z_{1} Z_{2} p_{3} \in$ $J$ and $\bar{f}^{2}-Y_{1} f=3\left(Z_{2}^{2} Z_{3}^{2}+\omega Z_{3}^{2} Z_{1}^{2}+\omega^{2} Z_{1}^{2} Z_{2}^{2}\right)$, we have

$$
9 \eta_{2}+v_{2} \eta_{1}-v_{0} v_{2}=0
$$

By (5.6), (5.10), we have

$$
3\left(\omega^{2}-\omega\right) \xi \equiv f^{3}-\bar{f}^{3} \equiv\left(v_{1} \eta_{1}+v_{3}-v_{0}\right) f \bar{f} \equiv\left(v_{1} \eta_{1}+v_{3}-v_{0}\right)\left(\eta_{1}^{2}-3 \eta_{3}\right)(\bmod J),
$$

hence

$$
3\left(\omega^{2}-\omega\right) \xi=\left(v_{1} \eta_{1}+v_{3}-v_{0}\right)\left(\eta_{1}^{2}-3 \eta_{3}\right)
$$

By the relation

$$
\begin{aligned}
v_{2} p_{3}- & \left(\omega-\omega^{2}\right)\left(\left(\omega^{2}+\omega v_{1}\right) Z_{1} p_{2}-\left(\omega+\omega^{2} v_{1}\right) Z_{2} p_{1}\right) \\
\equiv & v_{1}\left(9 \eta_{2}+v_{0} v_{1} v_{2}+v_{2} v_{3}-v_{0} v_{2}\right) Z_{3} \\
& +\left(3 v_{1} \eta_{1}+3 v_{3}-3 v_{1} v_{3}-v_{2}^{2}\right) Z_{1} Z_{2}(\bmod J)
\end{aligned}
$$


and $Z_{1} Z_{2}, Z_{3}$ representing two basis elements of $\mathbb{C}[Z] / J$, one obtains

$$
3 v_{1} \eta_{1}+3 v_{3}-3 v_{1} v_{3}-v_{2}^{2}=0, \quad v_{1}\left(9 \eta_{2}+v_{0} v_{1} v_{2}+v_{2} v_{3}-v_{0} v_{2}\right)=0
$$

With all the above relations among $v_{j}$ 's, $\eta_{k}$ 's, and $\xi$, one can conclude that $\left(v_{0}, v_{1}, v_{2}\right)$ forms a coordinate system of $\mathrm{Hilb}^{G}\left(\mathbb{C}^{3}\right)$ centered at $x_{\infty}$, and the other parameters in the expression of the ideal $J$ are expressed by the following relations:

$$
\begin{gathered}
v_{3}=\frac{1}{3} v_{2}^{2}-v_{0} v_{1}^{2}, \quad \eta_{1}=\frac{1}{3} v_{2}^{2}+v_{0} v_{1}-v_{0} v_{1}^{2}, \\
\eta_{2}=\frac{1}{27} v_{2}\left(3 v_{0}-3 v_{0} v_{1}-v_{2}^{2}+3 v_{0} v_{1}^{2}\right), \\
\eta_{3}=\frac{1}{27}\left(3 v_{0} v_{1}^{2}-v_{2}^{2}\right)\left(3 v_{0} v_{1}^{2}-3 v_{0} v_{1}-v_{2}^{2}+3 v_{0}\right), \\
\xi=\frac{\omega-\omega^{2}}{81} v_{0}\left(v_{1}+1\right)\left(3 v_{0} v_{1}^{2}+3 v_{0}-3 v_{0} v_{1}-v_{2}^{2}\right)\left(3 v_{0} v_{1}^{3}-v_{2}^{2}-v_{1} v_{2}^{2}\right)
\end{gathered}
$$

Note that the above $\xi, \eta_{1}, \eta_{2}, \eta_{3}$ satisfy relation (5.4). Furthermore, $v_{j}$ 's are $G$-invariant rational functions in $Z_{i}$ 's with the following expressions:

$$
\begin{gathered}
v_{0}=\frac{3\left(\omega-\omega^{2}\right) \xi-9 \eta_{1} \eta_{3}+27 \eta_{2}^{2}+2 \eta_{1}^{3}}{2\left(\eta_{1}^{2}-3 \eta_{3}\right)}, \quad v_{1}=\frac{\left(\omega-\omega^{2}\right) \xi+\eta_{1} \eta_{3}-9 \eta_{2}^{2}}{\left(\omega-\omega^{2}\right) \xi-\eta_{1} \eta_{3}+9 \eta_{2}^{2}} \\
v_{2}=\frac{6 \eta_{2}\left(\eta_{1}^{2}-3 \eta_{3}\right)}{\left(\omega-\omega^{2}\right) \xi-\eta_{1} \eta_{3}+9 \eta_{2}^{2}}, \quad v_{3}=\frac{-2 \eta_{3}\left(\eta_{1}^{2}-3 \eta_{3}\right)}{\left(\omega-\omega^{2}\right) \xi-\eta_{1} \eta_{3}+9 \eta_{2}^{2}} .
\end{gathered}
$$

This implies $d Z_{1} \wedge d Z_{2} \wedge d Z_{3}=\left(\left(\omega-\omega^{2}\right) / 36\right) d v_{0} \wedge d v_{1} \wedge d v_{2}$.

For $J$ near the element $x_{\infty}^{\prime}$ in $\operatorname{Hilb}^{G}\left(\mathbb{C}^{3}\right)$, we have

$$
J=\left\langle f^{2}-v_{0}^{\prime} \bar{f}, m_{6}-v_{1}^{\prime} m_{5}-v_{2}^{\prime} m_{4}-v_{3}^{\prime} m_{1}, Y_{1}-\eta_{1}, Y_{2}-\eta_{2}, Y_{3}-\eta_{3}, X-\xi\right\rangle .
$$

By an argument similar to the case $x_{\infty},\left(v_{0}^{\prime}, v_{1}^{\prime}, v_{2}^{\prime}\right)$ forms a coordinate system of $\operatorname{Hilb}^{G}\left(\mathbb{C}^{3}\right)$ centered at $x_{\infty}^{\prime}$ with the relations

$$
\begin{gathered}
v_{3}^{\prime}=\frac{1}{3} v_{2}^{\prime 2}-v_{0}^{\prime} v_{1}^{\prime 2}, \quad \eta_{1}=\frac{1}{3} v_{2}^{\prime 2}+v_{0}^{\prime} v_{1}^{\prime}-v_{0}^{\prime} v_{1}^{\prime 2}, \\
\eta_{2}=\frac{1}{27} v_{2}\left(3 v_{0}^{\prime}-3 v_{0}^{\prime} v_{1}^{\prime}-v_{2}^{\prime 2}+3 v_{0}^{\prime} v_{1}^{\prime 2}\right), \\
\eta_{3}=\frac{1}{27}\left(3 v_{0}^{\prime} v_{1}^{\prime 2}-v_{2}^{\prime 2}\right)\left(3 v_{0}^{\prime} v_{1}^{\prime 2}-3 v_{0}^{\prime} v_{1}^{\prime}-v_{2}^{\prime 2}+3 v_{0}^{\prime}\right), \\
\xi=\frac{\omega^{2}-\omega}{81} v_{0}^{\prime}\left(v_{1}^{\prime}+1\right)\left(3 v_{0}^{\prime} v_{1}^{\prime 2}+3 v_{0}^{\prime}-3 v_{0}^{\prime} v_{1}^{\prime}-v_{2}^{\prime 2}\right)\left(3 v_{0}^{\prime} v_{1}^{\prime 3}-v_{2}^{\prime 2}-v_{1}^{\prime} v_{2}^{\prime 2}\right)
\end{gathered}
$$


We have

$$
\begin{gathered}
v_{0}^{\prime}=\frac{3\left(\omega^{2}-\omega\right) \xi-9 \eta_{1} \eta_{3}+27 \eta_{2}^{2}+2 \eta_{1}^{3}}{2\left(\eta_{1}^{2}-3 \eta_{3}\right)}, \quad v_{1}^{\prime}=\frac{\left(\omega^{2}-\omega\right) \xi+\eta_{1} \eta_{3}-9 \eta_{2}^{2}}{\left(\omega^{2}-\omega\right) \xi-\eta_{1} \eta_{3}+9 \eta_{2}^{2}}, \\
v_{2}^{\prime}=\frac{6 \eta_{2}\left(\eta_{1}^{2}-3 \eta_{3}\right)}{\left(\omega^{2}-\omega\right) \xi-\eta_{1} \eta_{3}+9 \eta_{2}^{2}}, \quad v_{3}^{\prime}=\frac{-2 \eta_{3}\left(\eta_{1}^{2}-3 \eta_{3}\right)}{\left(\omega^{2}-\omega\right) \xi-\eta_{1} \eta_{3}+9 \eta_{2}^{2}},
\end{gathered}
$$

and $d Z_{1} \wedge d Z_{2} \wedge d Z_{3}=\left(\left(\omega^{2}-\omega\right) / 36\right) d v_{0}^{\prime} \wedge d v_{1}^{\prime} \wedge d v_{2}^{\prime}$.

For $J$ near $x_{0}$ in $\operatorname{Hilb}^{G}\left(\mathbb{C}^{3}\right)$, we have

$$
J=\left\langle f-u_{0} \bar{f}^{2}, \bar{m}_{9}-u_{1} m_{6}-u_{2} m_{4}-u_{3} m_{1}, Y_{1}-\eta_{1}, Y_{2}-\eta_{2}, Y_{3}-\eta_{3}, X-\xi\right\rangle,
$$

where $\left(\xi, \eta_{1}, \eta_{2}, \eta_{3}\right)$ is as before, and $\bar{m}_{9}-u_{1} m_{6}-u_{2} m_{4}-u_{3} m_{1}$ is the $G$-module $\sum_{j=1}^{3} \mathbb{C} q_{j}$ with $q_{1}:=\bar{f} Z_{2} Z_{3}-u_{1} \bar{f} Z_{1}-u_{2} Z_{2} Z_{3}-u_{3} Z_{1}, q_{2}:=\bar{f} \omega Z_{3} Z_{1}-u_{1} \bar{f} \omega Z_{2}-$ $u_{2} Z_{3} Z_{1}-u_{3} Z_{2}, q_{3}:=\bar{f} \omega^{2} Z_{1} Z_{2}-u_{1} \bar{f} \omega^{2} Z_{3}-u_{2} Z_{1} Z_{2}-u_{3} Z_{3}$. By the relation $-\left(u_{1}+\right.$ $\left.u_{0} u_{3}\right) \bar{f}^{2} \equiv-u_{1} \bar{f}^{2}-u_{3} f=Z_{1} q_{1}+\omega Z_{2} q_{2}+\omega^{2} Z_{3} q_{3}(\bmod J)$, we have

$$
u_{1}=-u_{0} u_{3} .
$$

By $\left(3 \eta_{2}-u_{1} \eta_{1}-u_{3}\right) \bar{f}=Z_{1} q_{1}+\omega^{2} Z_{2} q_{2}+\omega Z_{3} q_{3} \in J$, we have

$$
3 \eta_{2}=u_{1} \eta_{1}+u_{3}=u_{3}\left(1-u_{0} \eta_{1}\right) .
$$

By the relations $f^{2} \equiv u_{0} \bar{f}^{2} f \equiv u_{0}\left(\eta_{1}^{2}-3 \eta_{3}\right) \bar{f}(\bmod J)$,

$$
\begin{gathered}
Z_{2} Z_{3} q_{1}+\omega^{2} Z_{3} Z_{1} q_{2}+\omega Z_{1} Z_{2} q_{3} \equiv\left(\eta_{3}-3 u_{1} \eta_{2}\right) \bar{f}-u_{2}\left(Z_{2}^{2} Z_{3}^{2}+\omega^{2} Z_{3}^{2} Z_{1}^{2}+\omega Z_{1}^{2} Z_{2}^{2}\right)(\bmod J), \\
Z_{2}^{2} Z_{3}^{2}+\omega^{2} Z_{3}^{2} Z_{1}^{2}+\omega Z_{1}^{2} Z_{2}^{2} \equiv \frac{1}{3}\left(f^{2}-\eta_{1} \bar{f}\right) \equiv \frac{1}{3}\left(u_{0}\left(\eta_{1}^{2}-3 \eta_{3}\right)-\eta_{1}\right) \bar{f}(\bmod J),
\end{gathered}
$$

we have

$$
\left(1+u_{0} u_{2}\right) \eta_{3}=\frac{1}{3}\left(9 u_{1} \eta_{2}-u_{2} \eta_{1}+u_{0} u_{2} \eta_{1}^{2}\right) .
$$

Using (5.6), one has

$$
\begin{gathered}
u_{0} \bar{f}^{2} f^{2}-\bar{f}^{3} \equiv 3\left(\omega^{2}-\omega\right) \xi, \quad 2 u_{0}\left(\eta_{1}^{2}-3 \eta_{3}\right)^{2}-2 \bar{f}^{3} \equiv 6\left(\omega^{2}-\omega\right) \xi(\bmod J), \\
2 \bar{f}^{3} \equiv 27 \eta_{2}^{3}-9 \eta_{1} \eta_{3}+2 \eta_{1}^{3}-3\left(\omega^{2}-\omega\right) \xi(\bmod J),
\end{gathered}
$$

hence

$$
3\left(\omega^{2}-\omega\right) \xi=2 u_{0}\left(\eta_{1}^{2}-3 \eta_{3}\right)^{2}-27 \eta_{2}^{3}+9 \eta_{1} \eta_{3}-2 \eta_{1}^{3} .
$$

Using the above relations, we have

$$
\begin{aligned}
(1+ & \left.u_{0} u_{2}\right)\left(1-u_{0} \eta_{1}\right)\left(Z_{1} q_{2}-Z_{2} q_{1}-u_{1}\left(\omega-\omega^{2}\right) q_{3}\right) \\
& +\frac{1}{2+\omega}\left(1+u_{0} u_{2}\right)\left(-\omega^{2} u_{2} Z_{3}\left(f-u_{0} \bar{f}^{2}\right)+u_{0} u_{2} Z_{3}\left(f^{2}-u_{0}\left(\eta_{1}-3 \eta_{3}\right) \bar{f}\right)\right) \\
\equiv & \left(Z_{3} \bar{f}-Z_{3} \omega \eta_{1}\right)\left(1-u_{0} \eta_{1}\right)\left(\eta_{1}+u_{2}+u_{0} u_{2}^{2}-3 u_{0}^{2} u_{3}^{2}\right)(\bmod J) .
\end{aligned}
$$


As $Z_{3} \bar{f}, Z_{3}$ are two basis elements of $\mathbb{C}[Z] / J$, their coefficients in the last term of the above relation are zero. This implies

$$
\eta_{1}=-u_{2}-u_{0} u_{2}^{2}+3 u_{0}^{2} u_{3}^{2}
$$

From all the above relations between $u_{i}, \eta_{j}, \xi$, one concludes that $\left(u_{0}, u_{2}, u_{3}\right)$ forms a coordinate system of $\operatorname{Hilb}^{G}\left(\mathbb{C}^{3}\right)$ centered at $x_{0}$ and the following relations hold:

$$
\begin{gathered}
u_{1}=-u_{0} u_{3}, \quad \eta_{1}=-u_{2}-u_{0} u_{2}^{2}+3 u_{0}^{2} u_{3}^{2}, \\
\eta_{2}=\frac{1}{3} u_{3}\left(1+u_{0} u_{2}+u_{0}^{2} u_{2}^{2}-3 u_{0}^{3} u_{3}^{2}\right), \\
\eta_{3}=\frac{1}{3}\left(u_{2}^{2}-3 u_{0} u_{3}^{2}\right)\left(1+u_{0} u_{2}+u_{0}^{2} u_{2}^{2}-3 u_{0}^{3} u_{3}^{2}\right), \\
\xi=\frac{\omega-\omega^{2}}{9}\left(-1+u_{0} u_{2}\right)\left(3 u_{3}^{2}+u_{2}^{3}-3 u_{0} u_{2} u_{3}^{2}\right)\left(1+u_{0} u_{2}+u_{0}^{2} u_{2}^{2}-3 u_{0}^{3} u_{3}^{2}\right) .
\end{gathered}
$$

Again, the above expressions imply relation (5.4), and the $G$-invariant rational function expressions of $u_{i}$ 's are given as follows:

$$
\begin{aligned}
& u_{0}=\frac{6 \eta_{3}-2 \eta_{1}^{2}}{3\left(\omega^{2}-\omega\right) \xi+9 \eta_{1} \eta_{3}-2 \eta_{1}^{2}-27 \eta_{2}^{2}}, \\
& u_{1}=\frac{\eta_{2}\left(-6 \eta_{3}+2 \eta_{1}^{2}\right)}{\left(\omega^{2}-\omega\right) \xi+\eta_{1} \eta_{3}-9 \eta_{2}^{2}}, \\
& u_{2}=\frac{-\left(\omega^{2}-\omega\right) \xi \eta_{1}-6 \eta_{3}^{2}+9 \eta_{1} \eta_{2}^{2}+\eta_{1}^{2} \eta_{3}}{\left(\omega^{2}-\omega\right) \xi+\eta_{1} \eta_{3}-9 \eta_{2}^{2}}, \\
& u_{3}=\frac{\eta_{2}\left(3\left(\omega^{2}-\omega\right) \xi+9 \eta_{1} \eta_{3}-27 \eta_{2}^{2}-2 \eta_{1}^{3}\right)}{\left(\omega^{2}-\omega\right) \xi+\eta_{1} \eta_{3}-9 \eta_{2}^{2}},
\end{aligned}
$$

hence $d Z_{1} \wedge d Z_{2} \wedge d Z_{3}=\left(\left(\omega-\omega^{2}\right) / 12\right) d u_{0} \wedge d u_{2} \wedge d u_{3}$.

For $J$ near the element $x_{0}^{\prime}$ in $\operatorname{Hilb}^{G}\left(\mathbb{C}^{3}\right)$, we have

$$
J=\left\langle\bar{f}-u_{0}^{\prime} f^{2}, m_{9}-u_{1}^{\prime} m_{5}-u_{2}^{\prime} m_{4}-u_{3}^{\prime} m_{1}, Y_{1}-\eta_{1}, Y_{2}-\eta_{2}, Y_{3}-\eta_{3}, X-\xi\right\rangle .
$$

By an argument similar to the case $x_{0}$, one obtains that $\left(u_{0}^{\prime}, u_{2}^{\prime}, u_{3}^{\prime}\right)$ is an affine coordinate system with

$$
\begin{gathered}
u_{1}^{\prime}=-u_{0}^{\prime} u_{3}^{\prime}, \quad \eta_{1}=-u_{2}^{\prime}-u_{0}^{\prime} u_{2}^{\prime 2}+3 u_{0}^{\prime 2} u_{3}^{\prime 2}, \\
\eta_{2}=\frac{1}{3} u_{3}^{\prime}\left(1+u_{0}^{\prime} u_{2}^{\prime}+u_{0}^{\prime 2} u_{2}^{\prime 2}-3 u_{0}^{\prime 3} u_{3}^{\prime 2}\right), \\
\eta_{3}=\frac{1}{3}\left(u_{2}^{\prime 2}-3 u_{0}^{\prime} u_{3}^{\prime 2}\right)\left(1+u_{0}^{\prime} u_{2}^{\prime}+u_{0}^{\prime 2} u_{2}^{\prime 2}-3 u_{0}^{\prime 3} u_{3}^{\prime 2}\right), \\
\xi=\frac{\omega^{2}-\omega}{9}\left(-1+u_{0}^{\prime} u_{2}^{\prime}\right)\left(3 u_{3}^{\prime 2}+u_{2}^{\prime 3}-3 u_{0}^{\prime} u_{2}^{\prime} u_{3}^{\prime 2}\right)\left(1+u_{0}^{\prime} u_{2}^{\prime}+u_{0}^{\prime 2} u_{2}^{\prime 2}-3 u_{0}^{\prime 3} u_{3}^{\prime 2}\right),
\end{gathered}
$$


and the following relations hold:

$$
\begin{aligned}
& u_{0}^{\prime}=\frac{6 \eta_{3}-2 \eta_{1}^{2}}{3\left(\omega-\omega^{2}\right) \xi+9 \eta_{1} \eta_{3}-2 \eta_{1}^{2}-27 \eta_{2}^{2}}, \\
& u_{1}=\frac{\eta_{2}\left(-6 \eta_{3}+2 \eta_{1}^{2}\right)}{\left(\omega-\omega^{2}\right) \xi+\eta_{1} \eta_{3}-9 \eta_{2}^{2}}, \\
& u_{2}^{\prime}=\frac{-\left(\omega-\omega^{2}\right) \xi \eta_{1}-6 \eta_{3}^{2}+9 \eta_{1} \eta_{2}^{2}+\eta_{1}^{2} \eta_{3}}{\left(\omega-\omega^{2}\right) \xi+\eta_{1} \eta_{3}-9 \eta_{2}^{2}}, \\
& u_{3}^{\prime}=\frac{\eta_{2}\left(3\left(\omega-\omega^{2}\right) \xi+9 \eta_{1} \eta_{3}-27 \eta_{2}^{2}-2 \eta_{1}^{3}\right)}{\left(\omega-\omega^{2}\right) \xi+\eta_{1} \eta_{3}-9 \eta_{2}^{2}},
\end{aligned}
$$

hence $d Z_{1} \wedge d Z_{2} \wedge d Z_{3}=\left(\left(\omega^{2}-\omega\right) / 12\right) d u_{0}^{\prime} \wedge d u_{2}^{\prime} \wedge d u_{3}^{\prime}$.

With the analysis we have made in this section, one concludes that $\operatorname{Hilb}^{G}\left(\mathbb{C}^{3}\right)$ is covered by four affine spaces $\mathbb{C}^{3}$ centered at the central elements in Table 5.1, and the $G$-invariant volume form $d Z_{1} \wedge d Z_{2} \wedge d Z_{3}$ of $\mathbb{C}^{3}$ induces a never-vanishing global volume form of $\mathrm{Hilb}^{G}\left(\mathbb{C}^{3}\right)$. This completes the proof of Theorem 5.1.

6. Concluding remarks. In this paper, we have provided a detailed derivation of the smooth toric structure of $\operatorname{Hilb}^{A_{r}(4)}\left(\mathbb{C}^{4}\right)$. Its relation with crepant resolutions of $\mathbb{C}^{4} / A_{r}(4)$ has been found, and different crepant resolutions connected by flops of 4 folds can be visualized in the process. We have also given a constructive verification of the smooth and crepant properties of $\mathrm{Hilb}^{\mathfrak{A}_{4}}\left(\mathbb{C}^{3}\right)$ by a direct computation method. In the abelian case $A_{r}(4)$, the solution has been given in Sections 3 and 4 by the standard toric method, a combinatorial mechanism built upon monomials in $\mathbb{C}[Z]$, which can be regarded as characters of the whole torus group $T_{0}$, containing $A_{r}(4)$ as a finite subgroup. The smooth toric structure of $\operatorname{Hilb}^{A_{r}(4)}\left(\mathbb{C}^{4}\right)$ is derived from a procedure which mainly consists of two steps: first, one obtains a complete list of monomial ideals in $\mathrm{Hilb}^{A_{r}(4)}\left(\mathbb{C}^{4}\right)$ which correspond to the 0-dimensional toric orbits (see (3.13), (4.14), (4.20)); second, by the Gröbner basis technique and a detailed analysis of the $G$-regular module property of $\mathbb{C}[Z] / J$ for an ideal $J$ in $\operatorname{Hilb}^{A_{r}(4)}\left(\mathbb{C}^{4}\right)$, one proceeds to identify the toric coordinates from the ideal generators of $J$. In this manner, the explicit form of the canonical bundle of Hilb ${ }^{A_{r}(4)}\left(\mathbb{C}^{4}\right)$ can be determined as a disjoint sum of exceptional divisors, each of which could be blown down to give rise to crepant resolutions of $\mathbb{C}^{4} / A_{r}(4)$. These crepant resolutions are connected by a sequence of flops in 4 -folds through $\mathrm{Hilb}^{A_{r}(4)}\left(\mathbb{C}^{4}\right)$. We intend to apply a similar mechanism to the nonabelian case $G=\mathcal{A}_{n+1}$, but relying only on the data of $G$-representations in $\mathbb{C}[Z]$, a "big" group like the torus in the abelian case does not exist in the latter case though. In Section 6, we have made a detailed study on the structure of $\operatorname{Hilb}^{\mathfrak{X}_{4}}\left(\mathbb{C}^{3}\right)$, which would serve as a demonstration of the effectiveness of the method even though its crepant smooth conclusion is known by now [2]. We have succeeded to give an explicit verification of the crepant smooth structure of $\mathrm{Hilb}^{\mathfrak{H}_{4}}\left(\mathbb{C}^{3}\right)$, following our thought by a direct constructive method via group representations. An analysis similar to the higher-dimensional cases is now under progress and partial results are promising. As to the role of the $G$-Hilbert scheme in the study of crepant resolutions of $S_{G}$, our conclusion for the case $G=A_{r}(4)$ 
has indicated the noncrepant property of $\operatorname{Hilb}^{G}\left(\mathbb{C}^{4}\right)$, but with an intimate relation with crepant resolutions of $S_{G}$. For the higher-dimensional case, this kind of link between $\operatorname{Hilb}^{G}\left(\mathbb{C}^{n}\right)$ and some possibly existing crepant resolutions of $S_{G}$ could be further loosely related. However, the $G$-Hilbert scheme would still be worth further study in its own right due to the built-in character of group representations into the geometry of orbifolds. This could be a promising direction of the geometrical study of singularity. Such a program is now under our consideration for future study.

ACKNOWLEDGMENTS. This work was reported by the second author in the workshop "Modular Invariance, ADE, Subfactors and Geometry of Moduli Spaces," Kyoto, Japan, November 25-December 2, 2000, and as part of the subject of an Invited Lecture at the "7th International Symposium on Complex Geometry," Sugadaira, Japan, October 23-26, 2001, to which he is grateful for invitation and hospitality. The research of this paper was supported in part by the National Science Council of Taiwan under Grant no. 89-2115-M-001-037.

\section{REFERENCES}

[1] J. Bertin and D. Markushevich, Singularités quotients non abéliennes de dimension 3 et variétés de Calabi-Yau [Three-dimensional nonabelian quotient singularities and CalabiYau manifolds], Math. Ann. 299 (1994), no. 1, 105-116 (French).

[2] T. Bridgeland, A. King, and M. Reid, The McKay correspondence as an equivalence of derived categories, J. Amer. Math. Soc. 14 (2001), no. 3, 535-554.

[3] L. Chiang and S.-S. Roan, Orbifolds and finite group representations, Int. J. Math. Math. Sci. 26 (2001), no. 11, 649-669.

[4] D. Cox, J. Little, and D. O'Shea, Ideals, Varieties, and Algorithms, Undergraduate Texts in Mathematics, Springer-Verlag, New York, 1992.

[5] V. I. Danilov, The geometry of toric varieties, Uspekhi Mat. Nauk 33 (1978), no. 2(200), 85-134.

[6] Y. Gomi, I. Nakamura, and K. Shinoda, Hilbert schemes of G-orbits in dimension three, Asian J. Math. 4 (2000), no. 1, 51-70.

[7] Y. Ito and H. Nakajima, McKay correspondence and Hilbert schemes in dimension three, Topology 39 (2000), no. 6, 1155-1191.

[8] Y. Ito and I. Nakamura, McKay correspondence and Hilbert schemes, Proc. Japan Acad. Ser. A Math. Sci. 72 (1996), no. 7, 135-138.

[9] _ Hilbert schemes and simple singularities, New Trends in Algebraic Geometry (Warwick, 1996), London Math. Soc. Lecture Note Ser., vol. 264, Cambridge University Press, Cambridge, 1999, pp. 151-233.

[10] G. Kempf, F. F. Knudsen, D. Mumford, and B. Saint-Donat, Toroidal Embeddings. I, Lecture Notes in Mathematics, vol. 339, Springer-Verlag, Berlin, 1973.

[11] F. Klein, Gesammelte mathematische Abhandlungen, Springer-Verlag, Berlin, 1922, reprint 1973.

[12] G. A. Miller, H. F. Blichfeldt, and L. E. Dickson, Theory and Applications of Finite Groups, John Wiley \& Sons, New York, 1916.

[13] S. Mori, Birational classification of algebraic threefolds, Proceedings of the International Congress of Mathematicians, Vol. I, II (Kyoto, 1990), Mathematical Society of Japan, Tokyo, 1991, pp. 235-248.

[14] I. Nakamura, Hilbert Scheme and Simple Singularities $E_{6}, E_{7}$ and $E_{8}$, Preprint Series in Math., no. 362, Hokkaido University, Japan, 1996.

[15] _ Hilbert schemes of abelian group orbits, J. Algebraic Geom. 10 (2001), no. 4, 757779. 
[16] T. Oda, Torus Embeddings and Applications, Tata Institute of Fundamental Research Lectures on Mathematics and Physics, vol. 57, Tata Institute of Fundamental Research, Bombay, 1978.

[17] S.-S. Roan, Crepant resolution and fibred CY manifolds, Preprint MIAS 97-1, Institute of Mathematics, Academia Sinica, Taiwan, 1997.

[18] _ On the generalization of Kummer surfaces, J. Differential Geom. 30 (1989), no. 2, 523-537.

[19] _ Minimal resolutions of Gorenstein orbifolds in dimension three, Topology 35 (1996), no. $2,489-508$.

Li Chiang: Institute of Mathematics, Academia Sinica, Taipei 11529, Taiwan

E-mail address: chiang1@gate.sinica.edu.tw

Shi-Shyr Roan: Institute of Mathematics, Academia Sinica, Taipei 11529, Taiwan

E-mail address: maroan@ccvax.sinica.edu.tw 


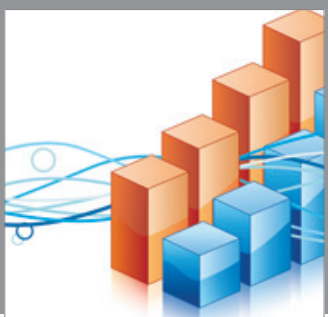

Advances in

Operations Research

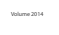

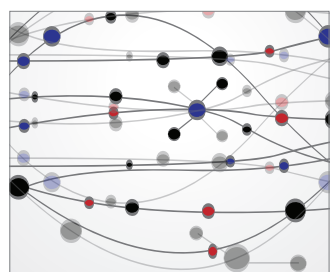

\section{The Scientific} World Journal
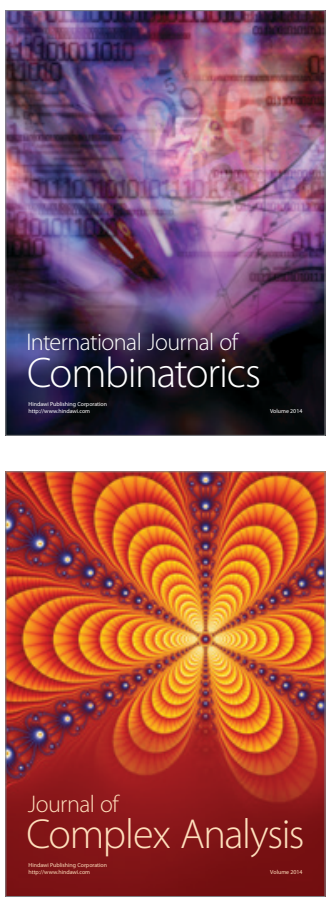

International Journal of

Mathematics and

Mathematical

Sciences
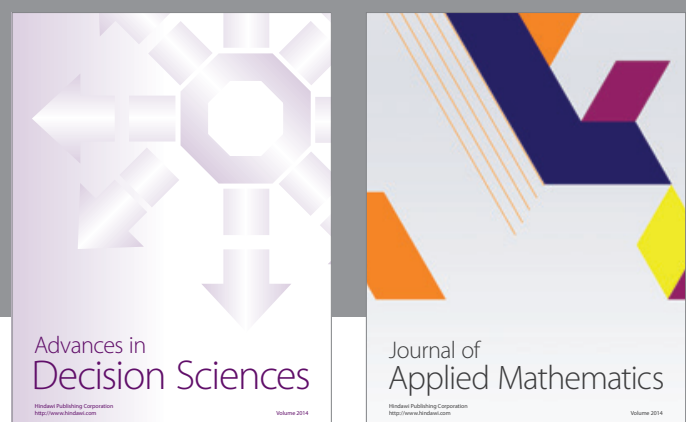

Journal of

Applied Mathematics
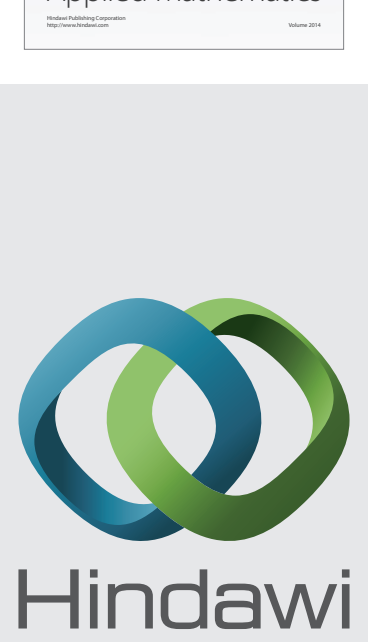

Submit your manuscripts at http://www.hindawi.com
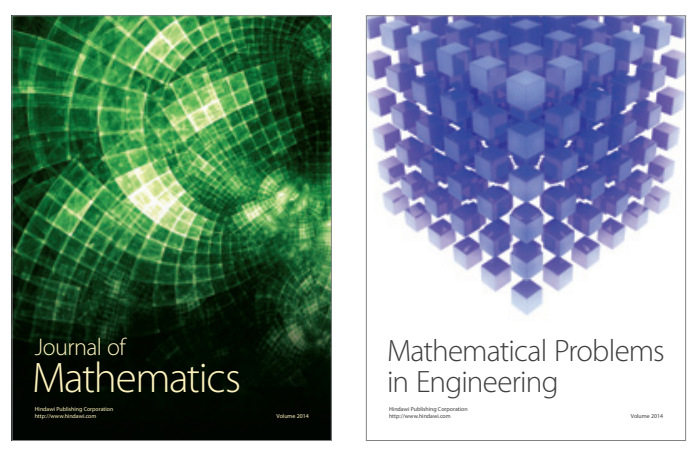

Mathematical Problems in Engineering
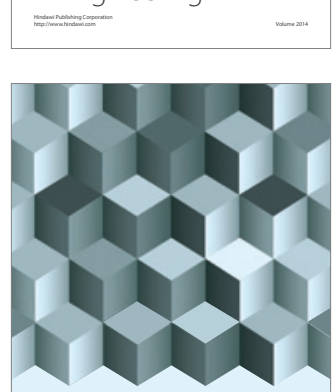

Journal of

Function Spaces
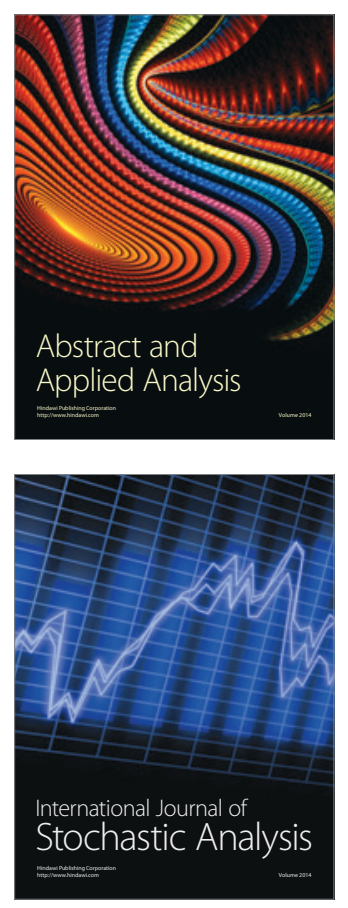

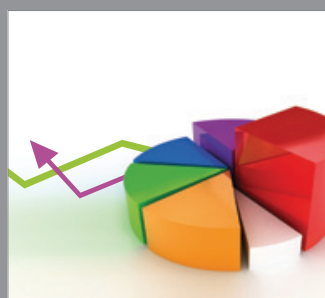

ournal of

Probability and Statistics

Promensencen
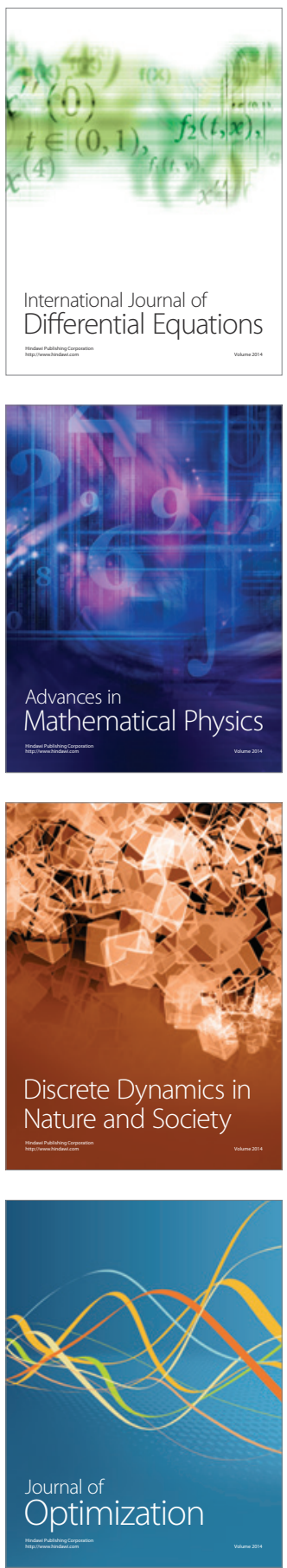